\title{
The Logic of Quasi-MV Algebras
}

\author{
FÉLIX BOU, Artificial Intelligence Research Institute (IIIA - CSIC), Bellaterra, \\ Spain.E-mail:fbou@iiia.csic.es
}

FRANCESCO PAOLI, Department of Education, University of Cagliari, Cagliari, Italy.E-mail:paoli@unica.it

ANTONIO LEDDA, Department of Education, University of Cagliari, Cagliari, Italy. E-mail: antonio.ledda@inwind.it

MATTHEW SPINKS, Mathematical Institute, University of Bern, Bern, Switzerland.E-mail: mspinksau@yahoo.com.au

ROBERTO GIUNTINI, Department of Education, University of Cagliari, Cagliari, Italy.E-mail: giuntini@unica.it

\begin{abstract}
The algebraic theory of quasi-MV algebras, generalizations of MV algebras arising in quantum computation, is by now rather well-developed. Although it is possible to define several interesting logics from these structures, so far this aspect has not been investigated. The present article aims at filling this gap.
\end{abstract}

Keywords: Quasi-MV algebras, quantum computation, MV algebras, abstract algebraic logic.

\section{Introduction}

Quasi-MV algebras were introduced in [20] in connection with quantum computation-namely, in an attempt to provide a convenient abstraction of the algebra over the set of all density operators of the Hilbert space $\mathbb{C}^{2}$, endowed with a suitable stock of quantum logical gates. Independently of their original quantum computational motivation, quasi-MV algebras present an additional, purely algebraic, motive of interest as generalizations of MV algebras to the semisubtractive (in the sense of [24]) but not point regular case. Later, $\sqrt{1}$ quasi-MV algebras were introduced as term expansions of quasi-MV algebras by an operation of square root of the inverse [17]. The above referenced papers contain the basics of the structure theory for these varieties, including appropriate standard completeness theorems w.r.t. the concrete algebras that constituted the motivational starting point of the whole investigation. Other algebraic works exploring quasi-MV algebras and $\sqrt{1}$ quasi-MV algebras are [6, 18, 19, 21].

Although it is possible to define several interesting logics from these structures, so far this aspect has not been investigated. The present article aims at filling this gap with respect to quasi-MV algebras. 


\subsection{Structure of the article}

The article is organized as follows. In Section 2, we present some preliminary material. Since we are especially interested in the logical aspects of this subject, it seems desirable to redefine the classes of algebras under investigation in such a way that implication is a primitive operation. Thus, we first introduce a term equivalent version of quasi-MV algebras, which we call quasi-Wajsberg algebras.

Next, we specify a general framework for the study of logics arising from preordered classes of algebras: we point out that each equationally definable preorder on a class of algebras gives rise to at least two interesting logics.

In Section 3, we apply this framework to logics arising from quasi-Wajsberg algebras. Besides the logical systems already known for Wajsberg algebras, we obtain in this way two new logics. In this section, we prove completeness results for each one of these logics. The results of this section are summarized in Table 2. In Section 4, we study these two new logics from the perspective of Abstract Algebraic Logic (AAL): we classify the deductive filters and the reduced matrices, we place the logics within the Leibniz hierarchy, etc. By so doing, we highlight the fact that several classes of quasi-Wajsberg algebras stand out for their logical significance. A summary of these classes is shown in Table 4.

In the interests of space, we omit the lenghty proof of Lemma 4, as well as the proof that the axioms of Definition 3 are independent. The web site accompanying this article [25] contains detailed proofs of these results.

Throughout the rest of the article, we assume on the reader's part some familiarity with the notation and content of at least the articles $[17,20]$. These remarks, notwithstanding, for the reader's convenience we shall briefly recall in Sections $2-4$ the most important definitions and results of $[17,20]$.

\section{Preliminaries}

\subsection{Notational conventions}

Throughout the present work, we will disregard the distinction between logical and algebraic languages, as is customary in AAL. If $\mathcal{L}$ is a language, its variables will be denoted either by the letters $p, q, r \ldots$ or by the letters $x, y, z \ldots$, whichever is more convenient. $\operatorname{Fm}(\mathcal{L})$ denotes the set of all formulas (terms) of $\mathcal{L}$. The letters $\alpha, \beta \ldots$ or $t, s \ldots$ are used as metavariables for arbitrary members of $\operatorname{Fm}(\mathcal{L})$. By $\alpha\left[p_{1}, \ldots, p_{n}\right]$ we mean that the set of variables occurring in $\alpha$ is a (proper or improper) subset of $\left\{p_{1}, \ldots, p_{n}\right\}$, while by $\alpha\left(p_{1} / \alpha_{1}, \ldots, p_{n} / \alpha_{n}\right)$ we indicate the result of replacing each $p_{i}$ in $\alpha$ by $\alpha_{i}$, possibly omitting the variables that get replaced by themselves. If $\mathbf{A}$ is an algebra and $a_{1}, \ldots, a_{n} \in A$, we denote the result of the application of the term operation $\alpha^{\mathbf{A}}$ to the elements $a_{1}, \ldots, a_{n}$ by $\alpha^{\mathbf{A}}\left(a_{1}, \ldots, a_{n}\right)$. We shall feel free to employ the self-explanatory vectorial notations $\alpha[\vec{p}]$ and $\alpha^{\mathbf{A}}(\vec{a})$ whenever the lengths of the indicated strings are clear from context. $\neg^{n} p$ stands for the variable $p$ preceded by $n$ occurrences $(n \geq 0)$ of the connective $\neg$.

Classes of algebras are denoted by means of blackboard bold letters. If a class is a singleton, outer brackets are dropped. Other notational conventions are as standard in universal algebra and AAL.

\subsection{Quasi-Wajsberg algebras}

MV algebras are usually presented, e.g. [8], in a language $\langle A, \oplus, \neg, 0\rangle$ of type $(2,1,0)$. However, considering implication as a primitive connective seems to better suit logical purposes. Therefore, 
whenever the study of logic is at issue, it is common to replace MV algebras by the term equivalent variety of Wajsberg algebras:

DEFINITION $1([8,15])$

A Wajsberg algebra is an algebra $\mathbf{A}=\langle A, \rightarrow, \neg, 1\rangle$ of type $(2,1,0)$ satisfying the following equations:

$$
\begin{aligned}
1 \rightarrow x & \approx x \\
(x \rightarrow y) \rightarrow((y \rightarrow z) \rightarrow(x \rightarrow z)) & \approx 1 \\
(x \rightarrow y) \rightarrow y & \approx(y \rightarrow x) \rightarrow x \\
(\neg x \rightarrow \neg y) \rightarrow(y \rightarrow x) & \approx 1
\end{aligned}
$$

We denote the class of Wajsberg algebras by $\mathbb{W}$.

It is well known that in any Wajsberg algebra, the other common MV operations can be defined as follows:

$$
\begin{array}{rlrl}
a \oplus b & :=\neg a \rightarrow b & a \odot b & :=\neg(\neg a \oplus \neg b) \\
a \ominus b & :=a \odot \neg b & a \vee b & :=(a \rightarrow b) \rightarrow b \\
a \wedge b & :=a \odot(a \rightarrow b) & 0 & :=\neg 1 \\
a \leftrightarrow b: & =(a \rightarrow b) \odot(b \rightarrow a) & &
\end{array}
$$

We now recall the definition of quasi-MV algebra.

DEFINITION 2 ([20])

A quasi-MV algebra is an algebra $\mathbf{A}=\langle A, \oplus, \neg, 1,0\rangle$ of type $(2,1,0,0)$ satisfying the following equations:

$$
\begin{aligned}
x \oplus(y \oplus z) & \approx(x \oplus z) \oplus y \\
\neg \neg x & \approx x \\
x \oplus 1 & \approx 1 \\
\neg(\neg x \oplus y) \oplus y & \approx \neg(\neg y \oplus x) \oplus x \\
\neg(x \oplus 0) & \approx \neg x \oplus 0 \\
(x \oplus y) \oplus 0 & \approx x \oplus y \\
\neg 0 & \approx 1
\end{aligned}
$$

From the definition it is clear that all MV algebras are quasi-MV algebras. On the other hand, we remind the reader that it is natural to think of a quasi-MV algebra as a generalization of an MV-algebra such that the equation $x \oplus 0 \approx x$ is not in general satisfied.

What is the 'logical' counterpart of quasi-MV algebras? Once again, it is desirable to have implication as a primitive connective. This leads us to the following. 
DEFINITION 3

A quasi-Wajsberg algebra is an algebra $\mathbf{A}=\langle A, \rightarrow, \neg, 1\rangle$ of type $(2,1,0)$ satisfying the following equations:

$$
\begin{aligned}
1 \rightarrow(x \rightarrow y) & \approx x \rightarrow y \\
(x \rightarrow y) \rightarrow((y \rightarrow z) \rightarrow(x \rightarrow z)) & \approx 1 \\
(x \rightarrow y) \rightarrow y & \approx(y \rightarrow x) \rightarrow x \\
(\neg x \rightarrow \neg y) \rightarrow(y \rightarrow x) & \approx 1 \\
\neg \neg x & \approx x \\
1 \rightarrow \neg(1 \rightarrow x) & \approx \neg(1 \rightarrow x)
\end{aligned}
$$

We denote the class of quasi-Wajsberg algebras by $\mathbf{q} \mathbb{W}$.

The independence of this axiomatization is shown in [25]. We notice that the last three equations in the presentation of Wajsberg algebras also trivially hold in quasi-Wajsberg algebras. Hence, the only Wajsberg axiom that can fail in quasi-Wajsberg algebras is (W1).

Before showing that quasi-Wajsberg algebras are term equivalent to quasi-MV algebras we will prove the following lemma.

LEMMA 4

Let $\mathbf{A}$ be a quasi-Wajsberg algebra. Then the following equations and quasiequations hold:

$$
\begin{aligned}
& x \rightarrow y \approx 1 \quad \& \quad y \rightarrow x \approx 1 \quad \Rightarrow \quad 1 \rightarrow x \approx 1 \rightarrow y \\
& x \rightarrow y \approx 1 \quad \& \quad y \rightarrow z \approx 1 \quad \Rightarrow \quad x \rightarrow z \approx 1 \\
& 1 \rightarrow 1 \approx 1 \\
& (1 \rightarrow x) \rightarrow(1 \rightarrow x) \approx 1 \\
& (x \rightarrow y) \rightarrow(x \rightarrow y) \approx 1 \\
& (1 \rightarrow x) \rightarrow x \approx 1 \\
& (1 \rightarrow x) \rightarrow 1 \approx 1 \\
& (x \rightarrow y) \rightarrow 1 \approx 1 \\
& ((x \rightarrow y) \rightarrow y) \rightarrow x \approx(x \rightarrow(y \rightarrow x)) \rightarrow(y \rightarrow x) \\
& (x \rightarrow(1 \rightarrow y)) \rightarrow(1 \rightarrow(x \rightarrow y)) \approx 1 \\
& (x \rightarrow y) \approx(\neg y \rightarrow \neg x) \\
& \neg(x \rightarrow y) \approx(x \rightarrow y) \rightarrow \neg 1 \\
& \neg 1 \approx 1 \rightarrow \neg 1 \\
& 1 \approx \neg 1 \rightarrow 1 \\
& (\neg(x \rightarrow y) \rightarrow \neg 1) \rightarrow(x \rightarrow y) \approx 1 \\
& ((x \rightarrow y) \rightarrow(\neg 1 \rightarrow(x \rightarrow y))) \rightarrow(\neg 1 \rightarrow(x \rightarrow y)) \approx 1 \\
& \neg 1 \rightarrow(x \rightarrow y) \approx 1 \\
& x \rightarrow 1 \approx 1 \\
& x \rightarrow(1 \rightarrow x) \approx 1 \\
& (1 \rightarrow x) \rightarrow y \approx x \rightarrow y
\end{aligned}
$$




$$
\begin{gathered}
x \rightarrow(1 \rightarrow y) \approx x \rightarrow y \\
(1 \rightarrow x) \rightarrow(1 \rightarrow y) \approx x \rightarrow y \\
x \rightarrow x \approx 1 \\
x \rightarrow(y \rightarrow x) \approx 1 \\
x \rightarrow(y \rightarrow z) \approx 1 \quad \Rightarrow \quad y \rightarrow(x \rightarrow z) \approx 1 \\
(x \rightarrow y) \rightarrow((z \rightarrow x) \rightarrow(z \rightarrow y)) \approx 1 \\
x \rightarrow(y \rightarrow z) \approx y \rightarrow(x \rightarrow z) \\
\neg 1 \rightarrow x \approx 1 \\
1 \rightarrow \neg x \approx x \rightarrow \neg 1 \\
1 \rightarrow \neg x \approx \neg(1 \rightarrow x)
\end{gathered}
$$

ProOF. See [25].

THEOREM 5 (Term equivalence)

(1) If $\mathbf{A}=\langle A, \rightarrow, \neg, 1\rangle$ is a quasi-Wajsberg algebra, then $f(\mathbf{A})=\langle A, \oplus, \neg, 1,0\rangle$, where $a \oplus b=\neg a \rightarrow$ $b$ and $0=\neg 1$, is a quasi-MV algebra.

(2) If $\mathbf{A}=\langle A, \oplus, \neg, 1,0\rangle$ is a quasi-MV algebra, then $g(\mathbf{A})=\langle A, \rightarrow, \neg, 1\rangle$, where $a \rightarrow b=\neg a \oplus b$, is a quasi-Wajsberg algebra.

(3) $f$ and $g$ are mutually inverse correspondences.

ProOF. The verifications needed to check the statements in the theorem are easy consequences of the properties given in the previous lemma and of known properties of quasi-MV algebras (see [20]).

\section{REMARK 6}

While it is possible to present Wajsberg algebras in the language $\langle\rightarrow, 0\rangle$ of type $(2,0)$ we point out that this is not the case for quasi-Wajsberg algebras, because $\neg$ cannot be defined using $\rightarrow$ and 0 . We notice that in general the equation $\neg x \approx x \rightarrow \neg 1$ does not hold for quasi-Wajsberg algebras.

Once we know the equivalences of the previous theorem, we can restate the known algebraic theorems for quasi-MV algebras in terms of quasi-Wajsberg algebras without providing any additional proof (see [6, 17, 20, 21] for the proofs). In the following, we will assume the definitions given above in (def) whenever we write any one of those symbols.

LEMMA 7 ([20])

Let $\mathbf{A}$ be a quasi-Wajsberg algebra. Then:

(1) The relation $\leq=\{\langle a, b\rangle \in A \times A: a \rightarrow b=1\}$ is a preorder. We denote the induced equivalence relation $\leq \cap \leq^{-1}$ by $\chi$.

(2) $a \chi b$ iff $1 \rightarrow a=1 \rightarrow b$.

(3) Every equivalence class of $\chi$ has one and only one element of the form $1 \rightarrow a$. These elements are said to be regular.

(4) $\chi$ is a congruence on any quasi-Wajsberg algebra $\mathbf{A}$.

(5) The set $R(A)$ of regular elements is the universe of a Wajsberg subalgebra of $\mathbf{A}$. Moreover, $\mathbf{R}(\mathbf{A}) \cong \mathbf{A} / \chi$. 
The class of flat quasi-Wajsberg algebras is the class of quasi-Wajsberg algebras that satisfy the additional equation $1 \approx \neg 1$. It is known that the class of flat algebras can also be characterized ${ }^{1}$ as the class of quasi-Wajsberg algebras satisfying $x \rightarrow y \approx 1$. An example of a flat algebra is the algebra $\mathbf{F}_{[\mathbf{0}, \mathbf{1}]}$ defined over $[0,1]$ with the following operations:

$$
a \rightarrow b:=\frac{1}{2} \quad \neg a:=1-a \quad 1:=\frac{1}{2}
$$

The notion of flat algebra helps us to give a characterization of quasi-Wajsberg algebras. ${ }^{2}$

THEOREM 8 ([20] Characterization of quasi-Wajsberg algebras)

(1) Every quasi-Wajsberg algebra A embeds into the direct product of a flat quasi-Wajsberg algebra $\mathbf{F}$ and a Wajsberg algebra $\mathbf{B}$. Moreover, we can choose $\mathbf{B}$ to be the subalgebra $\mathbf{R}(\mathbf{A})$ of regular elements in $\mathbf{A}$.

(2) The other direction also holds, i.e. all subalgebras of the direct product of a flat quasi-Wajsberg algebra and a Wajsberg algebra are quasi-Wajsberg algebras.

\section{Corollary 9}

A quasi-equation holds in the class of quasi-Wajsberg algebras iff it holds in flat quasi-Wajsberg algebras and in Wajsberg algebras.

In the case of Wajsberg algebras there is a distinguished algebra that is particularly interesting. It is called the standard Wajsberg algebra $\mathbf{S t}$ and is defined over the real unit interval $[0,1]$ with the following operations:

$$
\begin{array}{rlrl}
a \rightarrow b & :=\min \{1,1-a+b\} & \neg a & :=1-a \\
1 \quad & :=1 & 0 \quad:=0 \\
a \oplus b & :=\min \{1, a+b\} & a \odot b & :=\max \{0,1-(a+b)\} \\
a \ominus b & :=\max \{0, b-a\} & a \vee b & :=\max \{a, b\} \\
a \wedge b & :=\min \{a, b\} & a \leftrightarrow b & :=1-|b-a|
\end{array}
$$

Interest in the standard algebra comes from the fact that it generates the class of Wajsberg algebras as a quasivariety (see [16]). That is, a quasiequation holds in all Wajsberg algebras iff it holds in the standard one.

On the other hand, in [20], there are two proposals for the definition of a standard quasi-Wajsberg algebra. The first one is the square standard algebra $\mathbf{S}$ which is defined as the direct product of the flat algebra $\mathbf{F}_{[\mathbf{0}, \mathbf{1}]}$ and the Wajsberg algebra $\mathbf{S t}$. The second one is the disk standard algebra $\mathbf{D}$ which is defined as the subalgebra of $\mathbf{S}$ with universe

$$
\left\{\langle a, b\rangle \in[0,1] \times[0,1]:\left(a-\frac{1}{2}\right)^{2}+\left(b-\frac{1}{2}\right)^{2} \leq\left(\frac{1}{2}\right)^{2}\right\},
$$

i.e. the disk with centre $\left\langle\frac{1}{2}, \frac{1}{2}\right\rangle$ and radius $\frac{1}{2}$ in the complex plane.

\footnotetext{
${ }^{1}$ The non-trivial part can be proved in this way: $x \rightarrow y \stackrel{(\mathrm{qW} 1)}{=} 1 \rightarrow(x \rightarrow y) \stackrel{(\mathrm{qW} 17)}{=} \neg(x \rightarrow y) \rightarrow \neg 1 \stackrel{\text { Hypothesis }}{=} \neg(x \rightarrow y) \rightarrow$ $1 \stackrel{(\mathrm{qW} 24)}{=} 1$.

${ }^{2}$ In [6] we find a better characterization in terms of labelled Wajsberg algebras, but we will not need it in the present article. It is better in the sense that it characterizes all quasi-Wajsberg algebras up to isomorphism.
} 
THEOREM 10 ([20])

An equation holds in the class of quasi-Wajsberg algebras iff it holds in the square standard algebra. The same is also true for the disk standard algebra.

Although we can replace the word 'equation' by 'quasiequation' in the previous theorem in the case of Wajsberg algebras, we emphasize that this is not the case for quasi-Wajsberg algebras. For instance, ${ }^{3}$

- $0 \approx 1 \Rightarrow x \approx 1$ is valid both in $\mathbf{S}$ and in $\mathbf{D}$. However, it is not valid in all quasi-Wajsberg algebras.

- $x \approx 1 \& x \rightarrow y \approx 1 \Rightarrow y \approx 1$ is a valid quasiequation in $\mathbf{D}$. However, it is not valid in $\mathbf{S}$.

It is clear that quasi-Wajsberg algebras and Wajsberg algebras can be distinguished using an equation, e.g. $x \approx 1 \rightarrow x$. We will devote the rest of the section to proving that there is no way to distinguish quasi-Wajsberg algebras and Wajsberg algebras using equations of the form $t \approx 1$.

\section{DEFINITION 11}

A term $t$ in the language of quasi-Wajsberg algebras is called non-regular if it contains no occurrence of $\rightarrow$ nor of 1 . If it contains one of these symbols then the term is regular. ${ }^{4}$

We notice that non-regular terms are exactly the ones belonging to the set $\left\{\neg^{n} x\right.$ : $x$ is a variable and $n \in \omega\}$.

\section{LEMMA 12}

If $t$ is a regular term, then the equation $1 \rightarrow t \approx t$ is valid in all quasi-Wajsberg algebras.

PROOF. The proof is by induction on the construction of the term $t$. Let us analyse all cases.

- If $t$ is a variable then $t$ is non-regular. Hence, there is nothing to prove.

- If $t$ is the constant 1 , then we know that $1 \rightarrow t \approx t$ is valid by (qW9).

- If $t$ is $t_{1} \rightarrow t_{2}$, then we know that $1 \rightarrow t \approx t$ is valid by (qW1).

- Assume that $t$ is $\neg t_{1}$ and that $t$ is regular. Then, $t_{1}$ is also regular. Therefore, by the inductive hypothesis we know that $1 \rightarrow t_{1} \approx t_{1}$ is valid. Using (qW36) we conclude that $1 \rightarrow t \approx t$ is also valid.

The previous lemma tells us that regular terms in the sense of Definition 11 are exactly the regular elements in the sense of Lemma 7(3) in the free quasi-Wajsberg algebra with denumerably many generators.

\section{THEOREM 13}

If $t_{1}$ and $t_{2}$ are regular terms, then $t_{1} \approx t_{2}$ holds in all quasi-Wajsberg algebras iff it holds in all Wajsberg algebras.

Proof. Using Corollary 9 it is enough to prove that if $t_{1}$ and $t_{2}$ are regular terms, then $t_{1} \approx t_{2}$ is valid in all flat quasi-Wajsberg algebras. Therefore, as a consequence of Lemma 12 we know that it is enough to check that $1 \rightarrow t_{1} \approx 1 \rightarrow t_{2}$ holds in all flat quasi-Wajsberg algebras. And this is trivially true.

COROLlary 14

An equation $t \approx 1$ holds in all quasi-Wajsberg algebras iff it holds in all Wajsberg algebras.

\footnotetext{
${ }^{3}$ Observe that all equations appearing in these two quasiequations are of the form $t \approx 1$.

${ }^{4}$ This notion essentially corresponds to the concept of $\oplus$-normality, introduced in [6, Definition 18].
} 
PROOF. In case $t$ is a regular term the statement is a corollary of Theorem 13. If $t$ is not regular the claim is easily checked, because $t \approx 1$ fails in some Wajsberg algebra (and of course also in some quasi-Wajsberg algebra).

As a consequence of Theorem 10 and the completeness of the standard Wajsberg algebra, the previous corollary is saying that the classes $q \mathbb{W}, \mathbb{W}, \mathbf{S}, \mathbf{D}$ and St satisfy the same equations of the form $t \approx 1$.

We now recall a couple of notions from [21] that will turn out useful in the sequel. By $\mathbf{F}_{n m}$ we mean the finite flat algebra with $n$ fixpoints for the inverse (besides 0 ) and $m$ additional elements. Moreover, the Sand Glass is the finite quasi-Wajsberg algebra obtained from the 3-element Wajsberg chain by adjoining an element in the cloud of 1 and another element in the cloud of 0 . More precisely, the Sand Glass has $\left\{0, a, b, a^{\prime}, 1\right\}$ as a universe and the following tables for $\rightarrow$ and $\neg$ :

\begin{tabular}{l|lllll}
$\rightarrow$ & $\mathbf{0}$ & $\mathbf{a}$ & $\mathbf{b}$ & $\mathbf{a}^{\prime}$ & $\mathbf{1}$ \\
\hline $\mathbf{0}$ & 1 & 1 & 1 & 1 & 1 \\
$\mathbf{a}$ & 1 & 1 & 1 & 1 & 1 \\
$\mathbf{b}$ & $b$ & $b$ & 1 & 1 & 1 \\
$\mathbf{a}^{\prime}$ & 0 & 0 & $b$ & 1 & 1 \\
$\mathbf{1}$ & 0 & 0 & $b$ & 1 & 1
\end{tabular}

\begin{tabular}{l|l}
$\neg$ & \\
\hline $\mathbf{0}$ & 1 \\
$\mathbf{a}$ & $a^{\prime}$ \\
$\mathbf{b}$ & $b$ \\
$\mathbf{a}^{\prime}$ & $a$ \\
$\mathbf{1}$ & 0
\end{tabular}

\subsection{Logics from equationally preordered classes of algebras}

In this subsection, we introduce a general framework for defining logics from classes of algebras where at least one preorder relation is definable by means of equations. For every such preorder, at least two interesting logics arise: a logic whose valid inferences preserve the property of being evaluated as a designated maximal element (the top element, in case of partial orders); and a logic whose valid inferences are such that their conclusions are always evaluated above any element which is below the evaluations of all the premisses. If the elements of the algebras at issue are thought of as 'degrees of truth', in the spirit of some many-valued logics, the former logic can be seen as a logic of preservation of absolute truth, while the latter can be regarded as a logic of preservation of degrees of truth.

The following material is evidently indebted to the article [2], to which we owe the definition of equationally definable order. The main differences with the framework of Berman and Blok are essentially as follows:

- we generalize their definition to the case of preorders (that may not be partial orders);

- we allow for the case of preorder relations defined by means of parameterized formulae;

- while Berman and Blok's work is carried out in a purely algebraic setting, we adopt a logical setting in view of the particular applications to quantum computational logics we have in mind.

Although an investigation of the general theory of logics arising from equationally preordered classes of algebras is beyond the scope of this article, we will lay down some definitions here in order to accommodate the logics introduced in the next section within a framework which is as comprehensive as possible. Logics arising from such classes are studied in [22], where the concepts that follow are shown to have particular application to pointed varieties of algebras with a lattice term reduct. 


\section{DEFINITION 15}

Let $\mathbf{A}$ be an algebra of type $\sigma$ and let $P$ be a preorder on A. $P$ is called an equationally defined preorder for $\mathbf{A}$ iff there are $\sigma$-formulae $\alpha_{1}, \ldots, \alpha_{n}, \beta_{1}, \ldots, \beta_{n}$ of appropriate arities s.t., for any $a, b \in A$,

$$
\langle a, b\rangle \in P \text { iff for any } i \leq n \text {, and for any } \vec{c} \in A, \alpha_{i}^{\mathbf{A}}(a, b, \vec{c})=\beta_{i}^{\mathbf{A}}(a, b, \vec{c})
$$

In this case we also say that $\alpha_{1}, \ldots, \alpha_{n}, \beta_{1}, \ldots, \beta_{n}$ define the preorder $P$ on $\mathbf{A}$. If $\mathbb{K}$ is a class of algebras of type $\sigma$ and $\alpha_{1}, \ldots, \alpha_{n}, \beta_{1}, \ldots, \beta_{n}$ define a preorder $P$ on every algebra $\mathbf{A}$ in $\mathbb{K}$, we say that $P$ is an equationally defined preorder for $\mathbb{K}$.

\section{EXAMPLE 16}

The order induced by the lattice meet (or join) is an equationally defined preorder for the variety of lattices. Here we can choose $n=1, \alpha_{1}=p \wedge q, \beta_{1}=p$.

Henceforth, if $\leq$ is an equationally defined preorder for the class $\mathbb{K}$ of type $\sigma$ and $\varphi, \psi$ are $\sigma$ formulae, we will abbreviate by $\varphi \leq \psi$ the conjunction of equations

$$
\alpha_{1}(p / \varphi, q / \psi) \approx \beta_{1}(p / \varphi, q / \psi) \& \ldots \& \alpha_{n}(p / \varphi, q / \psi) \approx \beta_{n}(p / \varphi, q / \psi)
$$

Of course, there can be several different equationally defined preorders for the same class of algebras. For example, every discriminator variety of Boolean algebras with operators admits at least two distinct equationally definable partial orders (M. Spinks, R. J. Bignall, and R. Veroff, manuscript in preparation). Hereafter, we will confine ourselves to preorders that admit maximal elements.

\section{DEFINITION 17}

Let $\mathbb{K}$ be a class of algebras of type $\sigma$, and let $\leq$ be a fixed but arbitrary equationally defined preorder on $\mathbb{K}$. Further, let $\gamma_{1}, \ldots, \gamma_{n}, \alpha$ be $\sigma$-formulae, and let $c$ be a constant (which is added to $\sigma$ if it is not already present) denoting a designated maximal element of $\leq$ in any $\mathbf{A} \in \mathbb{K}$. We introduce two finitary logics:

- the truth-preserving logic $\vdash_{\mathbb{K}}^{\leq, c}$, defined by

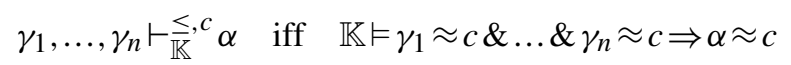

- the logic of degrees of truth $\vdash_{\mathbb{K}}^{\leq}$, defined by

$$
\gamma_{1}, \ldots, \gamma_{n} \vdash \underset{\mathbb{K}}{\leq} \alpha \quad \text { iff } \quad \mathbb{K} \vDash p \leq \gamma_{1} \& \ldots \& p \leq \gamma_{n} \Rightarrow p \leq \alpha
$$

If $\mathbb{K}$ is at least a quasivariety, then $\vdash_{\mathbb{K}}^{\leq, c}$ is nothing but the $c$-assertional logic of $\mathbb{K}$ [4]; when no equivocation is likely to arise, for the sake of notational irredundancy, we will write $\vdash_{\mathbb{K}}$ in place of $\vdash \leq \leq, c$.

\section{EXAMPLE 18}

Let $\mathbb{M V}$ be the variety of MV algebras, and let $\leq$ be the usual lattice order induced by the MV operations (choose $n=1, \alpha_{1}=1, \beta_{1}=p^{\prime} \oplus q$ ). Then $\vdash_{\mathbb{M V}}$ is Łukasiewicz infinite-valued logic, while $\vdash \leq \frac{\leq}{\mathbb{M V}}$ is the 'Eukasiewicz logic which preserves degrees of truth' of [12] (see also [5]). 
TABLE 1. Łukasiewicz logic $\mathbf{L}$ in the language $\rightarrow, \neg, 1$

\begin{tabular}{llll}
\hline (A1) & $\alpha \rightarrow(\beta \rightarrow \alpha)$ & (A2) & $(\alpha \rightarrow \beta) \rightarrow((\beta \rightarrow \gamma) \rightarrow(\alpha \rightarrow \gamma))$ \\
(A3) & $((\alpha \rightarrow \beta) \rightarrow \beta) \rightarrow((\beta \rightarrow \alpha) \rightarrow \alpha)$ & (A4) & $(\neg \alpha \rightarrow \neg \beta) \rightarrow(\beta \rightarrow \alpha)$ \\
(A5) & 1 & (MP) & $\alpha, \alpha \rightarrow \beta \vdash \beta$ \\
\hline
\end{tabular}

TABLE 2. Summary of the logic results

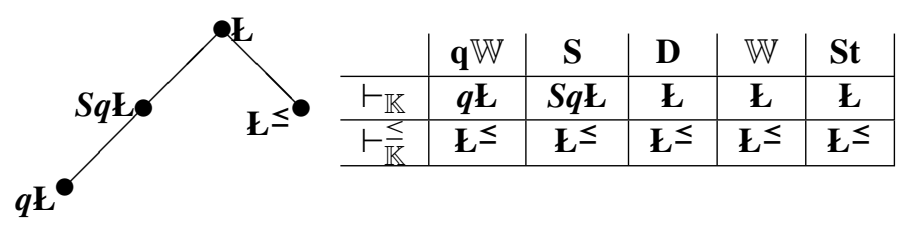

\section{Logical systems}

By Lemma 7, the relation $\leq$ is an equationally defined preorder for the variety of quasi-Wajsberg algebras (choose $n=1, \alpha_{1}=1, \beta_{1}=p \rightarrow q$ ). Therefore, according to Definition 17, quasi-Wajsberg algebras give rise both to a truth-preserving logic $\vdash_{\mathbf{q} \mathbb{W}}$ and to a logic of degrees of truth $\vdash_{\mathbf{q} \mathbb{W}}^{\leq}$. The same holds true for any subclass $\mathbb{K}$ of $\mathbf{q} \mathbb{W}$.

In the case of the class of Wajsberg algebras the logics obtained in this way are well known (cf. Example 18). The truth-preserving logic $\vdash_{\mathbb{W}}$ is nothing but the infinite-valued Łukasiewicz logic $\mathbf{E}$, which is presented in Table 1 (see [8]). On the other hand, the logic of degrees of truth $\vdash \underset{\mathbb{W}}{\leq}$ corresponds to the logic $\mathbf{L} \leq$, introduced in [26, Section 4.3] and deeply studied in the recent article [12]. In [12] it is proved that $\mathbf{L} \leq$ is characterized by the following two observations:

- $\mathbf{L} \leq$ has the same theorems as $\mathbf{L}$,

- the graded deduction-detachment theorem: $\alpha_{1}, \ldots, \alpha_{n} \vdash_{\mathbf{L}} \leq \alpha \quad$ iff $\quad \vdash_{\mathbf{L}} \leq\left(\alpha_{1} \wedge \ldots \wedge \alpha_{n}\right) \rightarrow \alpha$.

For the case of Wajsberg algebras it is well known that the logics defined above are exactly the ones we obtain if we consider just the standard Wajsberg algebra St.

In the rest of the section, we will axiomatize the truth-preserving logics and logics of degrees of truth obtained when we consider the following three classes of algebras: the class of quasi-Wajsberg algebras, the square standard algebra $\mathbf{S}$ and the disk standard algebra $\mathbf{D}$. The results we obtain are summarized in Table 2. Looking at this table, we see that we only need to introduce two new logical systems:

- The quasi-Łukasiewicz logic is the logical system $\mathbf{q \mathbf { L }}$ given in Table 3. We notice that this calculus has the same axioms as $\mathbf{L}$ and has the rules that we call quasi Modus Ponens (qMP), regularity (Reg), antiregularity (AReg1)/(AReg2)/(AReg3), involution (Inv1)/(Inv2) and flatness (Flat).

- The square quasi-Łukasiewicz logic $\mathbf{S q \mathbf { L }}$ is defined by the calculus for $\boldsymbol{q} \mathbf{E}$ augmented by the ex falso quodlibet rule

$$
\neg 1 \vdash \varphi \text {. }
$$

Next, we analyse in detail the first three columns of Table 2. 
TABLE 3. quasi-Łukasiewicz logic $\boldsymbol{q} \mathbf{L}$ in the language $\rightarrow, \neg, 1$

\begin{tabular}{llll}
\hline (A1) & $\alpha \rightarrow(\beta \rightarrow \alpha)$ & (A2) & $(\alpha \rightarrow \beta) \rightarrow((\beta \rightarrow \gamma) \rightarrow(\alpha \rightarrow \gamma))$ \\
(A3) & $((\alpha \rightarrow \beta) \rightarrow \beta) \rightarrow((\beta \rightarrow \alpha) \rightarrow \alpha)$ & (A4) & $(\neg \alpha \rightarrow \neg \beta) \rightarrow(\beta \rightarrow \alpha)$ \\
(A5) & 1 & (qMP) & $1 \rightarrow \alpha, 1 \rightarrow(\alpha \rightarrow \beta) \vdash 1 \rightarrow \beta$ \\
(Reg) & $\alpha \vdash 1 \rightarrow \alpha$ & (AReg1) & $1 \rightarrow(\alpha \rightarrow \beta) \vdash \alpha \rightarrow \beta$ \\
(AReg2) & $1 \rightarrow \neg(\alpha \rightarrow \beta) \vdash \neg(\alpha \rightarrow \beta)$ & (AReg3) & $1 \rightarrow \neg 1 \vdash \neg 1$ \\
(Inv1) & $\alpha \vdash \neg \neg \alpha$ & (Inv2) & $\neg \neg \alpha \vdash \alpha$ \\
(Flat) & $\alpha, \neg 1 \vdash \neg \alpha$ & & \\
\hline
\end{tabular}

\subsection{Quasi-Łukasiewicz logic $\mathbf{q} \mathbf{L}$}

In this subsection we will show that $\vdash_{\mathbf{q} \mathbb{W}}$ coincides with quasi-Łukasiewicz logic $\boldsymbol{q} \mathbf{E}$. The soundness of the calculus given in Table 3 is straightforward. Hence, we will devote the rest of the subsection to proving completeness. First of all, we state some properties of $\vdash_{\mathbf{q} \mathbb{W}}$.

LEMMA 19

Let $\alpha_{1}, \ldots, \alpha_{n}, \alpha$ be arbitrary formulas.

(1) The logic $\vdash_{\mathbf{q} \mathbb{W}}$ has the same theorems as $\vdash_{\mathbb{W}}$,

(2) if $\alpha_{1}, \ldots, \alpha_{n} \vdash_{\mathbf{q} \mathbb{W}} \alpha$, then $\alpha_{1}, \ldots, \alpha_{n} \vdash_{\mathbb{W}} \alpha$,

(3) $\alpha_{1}, \ldots, \alpha_{n} \vdash_{\mathbb{W}} \alpha$ iff $\quad \alpha_{1}, \ldots, \alpha_{n} \vdash_{\mathbf{q} \mathbb{W}} 1 \rightarrow \alpha$,

(4) $\alpha_{1}, \ldots, \alpha_{n} \vdash_{\mathbb{W}} 0$ iff $\alpha_{1}, \ldots, \alpha_{n} \vdash_{\mathbf{q} \mathbb{W}} 0$,

(5) if $\alpha$ is a regular formula, then $\alpha_{1}, \ldots, \alpha_{n} \vdash \mathbb{W} \alpha$ iff $\alpha_{1}, \ldots, \alpha_{n} \vdash_{\mathbf{q} \mathbb{W}} \alpha$.

PROOF. The first part is a consequence of Corollary 14. The second item is a consequence of the fact that all Wajsberg algebras are quasi-Wajsberg algebras. The last two items are easy consequences of the third item, using (qW19) and Lemma 12. The third item is easily proved, thanks to the fact that the subalgebra of regular elements of any quasi-Wajsberg algebra is a Wajsberg algebra.

The next proposition, which characterizes the inferences of $\vdash_{\mathbf{q} \mathbb{W}}$ according to the logical form of their conclusions, is the key to our completeness proof. In the statement of the proposition and in the sequel, the parity of a natural number is either odd or even.

PROPOSITION 20 (Characterization of $\vdash_{\mathbf{q} \mathbb{W}}$ )

Let $\alpha_{1}, \ldots, \alpha_{n}, \alpha$ be arbitrary formulas. Then, $\alpha_{1}, \ldots, \alpha_{n} \vdash_{\mathbf{q} \mathbb{W}} \alpha$ iff at least one of the following conditions holds:

(1) $\alpha$ is a regular formula and $\alpha_{1}, \ldots, \alpha_{n} \vdash \mathbb{w} \alpha$,

(2) there is a variable $p$ and two natural numbers $m, k$ with the same parity such that $\alpha=\neg^{m} p$ and $\neg^{k} p \in\left\{\alpha_{1}, \ldots, \alpha_{n}\right\}$

(3) there is a variable $p$, and two natural numbers $m$ and $k$ with different parities such that $\alpha=\neg^{m} p$, $\neg^{k} p \in\left\{\alpha_{1}, \ldots, \alpha_{n}\right\}$ and $\alpha_{1}, \ldots, \alpha_{n} \vdash \mathbb{W} 0$.

PROOF. It is easy to check that any of these three properties implies $\alpha_{1}, \ldots, \alpha_{n} \vdash_{\mathbf{q} \mathbb{W}} \alpha$. The reader can avail him or herself of Lemma 19 and the involutive and flatness rules of $\boldsymbol{q} \mathbf{E}$.

For the other direction, assume that $\alpha_{1}, \ldots, \alpha_{n} \vdash_{\mathbf{q} \mathbb{W}} \alpha$. We distinguish three cases according to the shape of the formula $\alpha$.

Case $\alpha$ is regular: Then, Lemma 19 implies that Condition 1 obtains.

Case $\alpha$ is $\neg^{m} p$ with $p$ a variable and $m$ an even number: Here we consider two subcases.

Subcase $\left\{\neg^{k} p: k\right.$ even $\} \cap\left\{\alpha_{1}, \ldots, \alpha_{n}\right\} \neq \emptyset$ : Then, it is obvious that Condition 2 obtains. 
Subcase $\left\{\neg^{k} p: k\right.$ even $\} \cap\left\{\alpha_{1}, \ldots, \alpha_{n}\right\}=\emptyset$ : In this subcase we are going to see that Condition 3 obtains. We define $X, Y$ as follows:

$$
\begin{gathered}
X=\left\{q: \text { there exists an even } k \in \omega \text { such that } \neg^{k} q \in\left\{\alpha_{1}, \ldots, \alpha_{n}\right\}\right\} \\
Y=\left\{q: q \notin X \cup\{p\} \text { and there exists an odd } k \in \omega, \neg^{k} q \in\left\{\alpha_{1}, \ldots, \alpha_{n}\right\}\right\} .
\end{gathered}
$$

Owing to the hypotheses of this subcase, it is clear that $p \notin X$. And it is also clear that $X \cap Y=\emptyset$ and $p \notin Y$. Then, for every formula $\gamma$, we define $\tilde{\gamma}$ as the result of replacing the variables in $X$ by 1 and the variables in $Y$ by 0 . Since $\alpha_{1}, \ldots, \alpha_{n} \vdash_{\mathbf{q} \mathbb{W}} \alpha$ we deduce that $\widetilde{\alpha_{1}}, \ldots, \widetilde{\alpha_{n}} \vdash_{\mathbf{q} \mathbb{W}} \widetilde{\alpha}$. We notice that since $p \notin X \cup Y$, it holds that $\widetilde{\alpha}=\alpha=\neg^{m} p$.

First of all, let us see that there is an $i$ such that $\tilde{\alpha}_{i}$ is not regular. For suppose otherwise. Then, all $\widetilde{\alpha_{1}}, \ldots, \tilde{\alpha_{n}}$ are regular, and by the proof of Theorem 13 we know that for every $j$, $\tilde{\alpha_{j}} \approx 1$ is valid in all flat quasi-Wajsberg algebras. Hence, it is easy to see that $\tilde{\alpha_{1}} \approx 1 \& \ldots \&$ $\widetilde{\alpha_{n}} \approx 1 \Rightarrow \neg^{m} p \approx 1$ fails in some quasi-Wajsberg algebra. Therefore, $\tilde{\alpha_{1}}, \ldots,{\tilde{\alpha_{n}}}_{n} \forall_{\mathbf{q} \mathbb{W}} \neg^{m} p$, a contradiction.

Hence, we can assume that there is an $i$ such that $\widetilde{\alpha_{i}}$ is not regular. Thus, $\tilde{\alpha}_{i}$ is of the form $\neg^{k} q$ for some variable $q$ and some number $k$. By the construction of $\widetilde{\alpha_{i}}$ this implies that $\alpha_{i}=\neg^{k} p$ and that $q=p$. By the hypotheses of this subcase it follows that $k$ is odd.

Therefore, the only property of Condition 3 that remains to be established is that $\alpha_{1}, \ldots, \alpha_{n} \vdash_{\mathbb{W}} 0$. For this it is enough to check that $\alpha_{1}, \ldots, \alpha_{n} \vdash_{\mathbb{W}} \alpha$ and that $\alpha_{1}, \ldots, \alpha_{n} \vdash_{\mathbb{W}} \neg \alpha$. The first part follows from Lemma 19(2). And the second part follows on observing that $\alpha_{i}=\neg^{k} p$ with $k$ odd and that $\alpha=\neg^{m} p$ with $m$ even.

Case $\alpha$ is $\neg^{m} p$ with $p$ a variable and $m$ an odd number: This case is symmetric to the previous one.

THEOREM 21 (Completeness of quasi-Łukasiewicz logic)

Let $\alpha_{1}, \ldots, \alpha_{n}, \alpha$ be arbitrary formulas. Then,

$$
\alpha_{1}, \ldots, \alpha_{n} \vdash_{\mathbf{q} \mathbf{E}} \alpha \quad \text { iff } \quad \alpha_{1}, \ldots, \alpha_{n} \vdash_{\mathbf{q} \mathbb{W}} \alpha .
$$

PROOF. The soundness part is straightforward. For completeness we need some claims.

CLAIM 22

If $\alpha_{1}, \ldots, \alpha_{n} \vdash_{\mathbf{L}} \alpha$ then $\alpha_{1}, \ldots, \alpha_{n} \vdash_{q \mathbf{L}} 1 \rightarrow \alpha$.

Proof Of THE Claim. The proof is a simple induction on the length of the derivation of $\alpha$ from $\alpha_{1}, \ldots, \alpha_{n}$ in $\mathbf{E}$. The case of the axioms follows from the regularity rule (Reg). The case involving the Modus Ponens rule follows from the qMP rule.

\section{Claim 23}

If $\alpha$ is a regular formula then $1 \rightarrow \alpha \vdash_{q \mathbf{L}} \alpha$.

Proof of the Claim. To establish the claim it suffices to prove via (simultaneous) induction on the formula $\alpha$ that the following two conditions hold:

- if $\alpha$ is regular then $1 \rightarrow \alpha \vdash_{q \mathbf{E}} \alpha$,

- if $\alpha$ is regular then $1 \rightarrow \neg \alpha \vdash_{q \mathbf{L}} \neg \alpha$.

Let us consider the different cases that appear in the induction. In case $\alpha$ is a variable there is nothing to be proved, because the antecedents are false. If $\alpha$ is 1 our claims are easily proved. The 
case that $\alpha$ is an implication follows from the antiregularity rules. We consider in some detail the case that $\alpha$ is the negation of some formula $\beta$ and $\alpha$ is regular. Then, $\beta$ is also regular. Therefore, by the inductive hypothesis we know that $1 \rightarrow \beta \vdash_{\boldsymbol{q} \mathbf{E}} \beta$ and that $1 \rightarrow \neg \beta \vdash_{\boldsymbol{q \mathbf { L }}} \neg \beta$. Using $1 \rightarrow \neg \neg \beta \vdash_{\mathbf{L}} \beta$ and Fact 22 we know that $1 \rightarrow \neg \neg \beta \vdash_{q \mathbf{L}} 1 \rightarrow \beta$. Hence, by the inductive hypothesis and the involution rule we conclude that $1 \rightarrow \neg \neg \beta \vdash_{\mathbf{q L}} \neg \neg \beta$.

Claim 24

If $\alpha_{1}, \ldots, \alpha_{n} \vdash_{\mathbf{L}} 0$ then $\alpha_{1}, \ldots, \alpha_{n} \vdash_{q \mathbf{L}} 0$.

ProOf OF THE Claim. This a consequence of Claim 22 and the rule (AReg3).

Now we are ready to prove the completeness part. Assume that $\alpha_{1}, \ldots, \alpha_{n} \vdash_{\mathbf{q} \mathbb{W}} \alpha$. We remind the reader that $\mathbf{L}$ is complete with respect to $\vdash_{\mathbb{W}}$. By Proposition 20, one of the Conditions 1-3 holds. Let us consider them one by one.

- In the first case we obtain that $\alpha_{1}, \ldots, \alpha_{n} \vdash_{\boldsymbol{q} \mathbf{E}} \alpha$ by Claims 22 and 23.

- In the second case we obtain that $\alpha_{1}, \ldots, \alpha_{n} \vdash_{q \mathbf{E}} \alpha$ using the involutive rules.

- In the third case we obtain that $\alpha_{1}, \ldots, \alpha_{n} \vdash_{\mathbf{q} \mathbf{E}} \alpha$ using Claim 24, the involutive rules and the flatness rule.

In the definition of $\vdash_{\mathbf{q} \mathbb{W}}$ we have used a large class of algebras, all quasi-Wajsberg algebras. It is natural to wonder whether it is enough to consider a single quasi-Wajsberg algebra. Unfortunately, the next proposition shows this is false. In particular, this means that there is no way to prove the completeness of $\vdash_{\mathbf{q} \mathbb{W}}$ by building a Lindenbaum-Tarski algebra.

\section{PROPOSITION 25}

There is no quasi-Wajsberg algebra $\mathbf{A}$ such that $\boldsymbol{q} \mathbf{L}$ coincides with $\vdash_{\mathbf{A}}$.

PROOF. It is enough to observe that $0 \forall_{\mathbf{q} \mathbf{L}} p$ and that $p \nvdash_{\mathbf{q} \mathbf{\mathbf { L }}} \neg p$. This suffices because $0 \nvdash_{\mathbf{A}} p$ implies that $\mathbf{A}$ is a flat quasi-Wajsberg algebra, while $p \nvdash_{\mathbf{A}} \neg p$ implies that $\mathbf{A}$ is not a flat quasi-Wajsberg algebra.

\subsection{Square Quasi-Łukasiewicz logic $\mathbf{S q \mathbf { L }}$}

In this subsection, we will show that $\vdash_{\mathbf{S}}$ coincides with the square quasi-Łukasiewicz logic $S q \mathbf{L}$. We notice that since $\mathbf{S}$ is a quasi-Wajsberg algebra we already know that $\vdash_{\mathbf{q} \mathbb{W}} \leq \vdash_{\mathbf{S}}$.

The proof of the completeness is very similar to the one given in the previous subsection. This is because we have the following characterization of $\vdash_{\mathbf{S}}$.

Proposition 26 (Characterization of $\vdash_{\mathbf{s}}$ )

Let $\alpha_{1}, \ldots, \alpha_{n}, \alpha$ be arbitrary formulas. Then, $\alpha_{1}, \ldots, \alpha_{n} \vdash_{\mathbf{S}} \alpha$ iff at least one of the following conditions holds

(1) $\alpha$ is a regular formula and $\alpha_{1}, \ldots, \alpha_{n} \vdash_{\mathbb{W}} \alpha$,

(2) there is a variable $p$ and two natural numbers $m, k$ with the same parity such that $\alpha=\neg^{m} p$ and $\neg^{k} p \in\left\{\alpha_{1}, \ldots, \alpha_{n}\right\}$

(3) $\alpha_{1}, \ldots, \alpha_{n} \vdash_{\mathbb{W}} 0$.

Proof. First of all we notice that the first two conditions appear in Proposition 20.

Using the rule (EFQ) and the fact that $q \mathbf{L} \leq S q \mathbf{L}$ it is obvious that any of these three properties implies that $\alpha_{1}, \ldots, \alpha_{n} \vdash \mathbb{W} \alpha$. 
For the other direction we can argue as in Proposition 20. The only difference is that, for the counterexample required by the argument, we need to use the flat algebra $\mathbf{F}_{[\mathbf{0}, \mathbf{1}]}$ (introduced after Lemma 7) and not an arbitrary flat quasi-Wajsberg algebra.

THEOREM 27 (Completeness of square quasi-Łukasiewicz logic) Let $\alpha_{1}, \ldots, \alpha_{n}, \alpha$ be arbitrary formulas. Then,

$$
\alpha_{1}, \ldots, \alpha_{n} \vdash_{S q \mathbf{L}} \alpha \quad \text { iff } \quad \alpha_{1}, \ldots, \alpha_{n} \vdash_{\mathbf{S}} \alpha .
$$

Proof. The soundness part is straightforward. For completeness let us assume that $\alpha_{1}, \ldots, \alpha_{n} \vdash_{\mathbf{S}} \alpha$. By Proposition 26, we know that one of Conditions 1-3 obtains. In the first two cases $\vdash_{q \mathbf{E}} \leq \vdash_{S q \mathbf{E}}$ implies that $\alpha_{1}, \ldots, \alpha_{n} \vdash \mathbf{S q \mathbf { E }} \alpha$. And in the third case we can prove that $\alpha_{1}, \ldots, \alpha_{n} \vdash_{S q \mathbf{E}} \alpha$ thanks to the rule (EFQ) and Claim 24.

\subsection{Eukasiewicz $\operatorname{logic} \mathbf{t}$}

The aim of this subsection is to show that infinite-valued Łukasiewicz logic is complete with respect to the truth-preserving logic $\vdash_{\mathbf{D}}$ associated with the disk standard algebra $\mathbf{D}$. In this case the proof is very simple.

THEOREM 28 (Completeness)

Let $\alpha_{1}, \ldots, \alpha_{n}, \alpha$ be arbitrary formulas. Then,

$$
\alpha_{1}, \ldots, \alpha_{n} \vdash_{\mathbf{L}} \alpha \quad \text { iff } \quad \alpha_{1}, \ldots, \alpha_{n} \vdash_{\mathbf{D}} \alpha .
$$

ProOF. It is straightforward to verify that the postulates of $\mathbf{L}$ are sound for $\vdash_{\mathbf{D}}$. Hence, $\vdash_{\mathbf{L}} \leq \vdash_{\mathbf{D}}$.

On the other hand, since the subalgebra of regular elements of $\mathbf{D}$ is isomorphic to the standard Wajsberg algebra $\mathbf{S t}$, it follows that $\vdash_{\mathbf{D}} \leq \vdash_{\mathbf{S t}}$. Finally using the completeness of $\mathbf{L}$ with respect to the standard Wajsberg algebra we obtain $\vdash_{\mathbf{D}} \leq \vdash_{\mathbf{L}}$.

Finally, we notice that it is very easy to see that $\mathbf{L}$ coincides with the logic given by the calculus of $\boldsymbol{q} \mathbf{L}$ extended by the following antiregularity rule

$$
1 \rightarrow \alpha \vdash \alpha
$$

\subsection{The logic $\mathbf{L} \leq$}

The aim of this subsection is to prove that

$$
\vdash_{\mathbf{q} \mathbb{W}}^{\leq}=\vdash \frac{\leq}{\mathbf{S}}=\vdash_{\mathbf{D}}^{\leq}=\vdash_{\mathbb{W}}^{\leq}=\vdash_{\mathbf{S t}}^{\leq} .
$$

First of all we observe that, if $\mathbb{K}$ is a class of quasi-Wajsberg algebras, then $\vdash_{\mathbb{K}}^{\leq}$has a graded deduction-detachment theorem in the form

$$
\alpha_{1}, \ldots, \alpha_{n} \vdash \underset{\mathbb{K}}{<} \alpha \quad \text { iff } \quad \mathbb{K} \text { satisfies }\left(\alpha_{1} \wedge \cdots \wedge \alpha_{n}\right) \rightarrow \alpha \approx 1 .
$$

This follows because $p \rightarrow\left(\alpha_{1} \wedge \ldots \wedge \alpha_{n}\right) \approx\left(p \rightarrow \alpha_{1}\right) \wedge \ldots \wedge\left(p \rightarrow \alpha_{n}\right)$ holds in all quasi-Wajsberg algebras (see Corollary 9 ). Using this remark it is easy to prove the following result. 
THEOREM 29 (Completeness)

Let $\alpha_{1}, \ldots, \alpha_{n}, \alpha$ be arbitrary formulas. The following statements are equivalent:

(1) $\alpha_{1}, \ldots, \alpha_{n} \vdash_{\mathbf{L}} \leq \alpha$,

(2) $\alpha_{1}, \ldots, \alpha_{n} \vdash \underset{\mathbf{q} \mathbb{W}}{\leq} \alpha$,

(3) $\alpha_{1}, \ldots, \alpha_{n} \vdash \frac{\leq}{\mathbf{S}} \alpha$

(4) $\alpha_{1}, \ldots, \alpha_{n} \vdash \frac{\leq}{\mathbf{D}} \alpha$.

PROOF. The preceding observation and Corollary 14 imply the equivalence between the first two statements.

Obviously, $\vdash_{\mathbf{L}} \leq \leq \vdash_{\mathbf{S}}^{\leq}$and $\vdash_{\mathbf{L}} \leq \leq \vdash \frac{\leq}{\mathbf{D}}$. On the other hand, since the subalgebras of regular elements

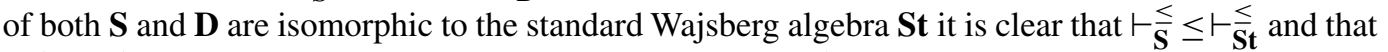

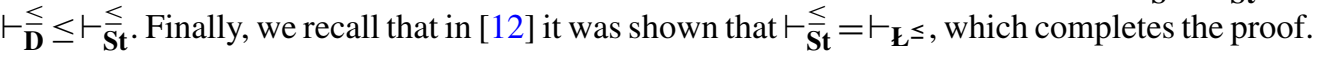

\subsection{Comparison between the logics}

The aim of this subsection is to prove that the graph depicted in Table 2 accurately describes the inclusion relationships between our four logics.

PROPOSITION 30

The following statements hold:

(1) $\mathbf{L} \leq<\mathbf{L}$,

(2) $\boldsymbol{q} \mathbf{L}<\boldsymbol{S q \mathbf { L }}<\mathbf{L}$,

(3) $\boldsymbol{q} \mathbf{L}$ and $\mathbf{E} \leq$ are incomparable,

(4) $S q \mathbf{L}$ and $\mathbf{E} \leq$ are incomparable.

Proof. (1) This was proved in [12].

(2) From our calculi it is obvious that $\boldsymbol{q} \mathbf{L} \leq \boldsymbol{S q \mathbf { L }} \leq \mathbf{\mathbf { L }}$. These inclusions are strict since (i) $0 \nvdash_{\boldsymbol{q} \mathbf{E}} p$, (ii) $0 \vdash_{S q \mathbf{L}} p$, (iii) $1 \rightarrow p \nvdash_{S q \mathbf{L}} p$ and (iv) $1 \rightarrow p \vdash_{\mathbf{L}} p$.

(3) This follows on observing (i) $1 \rightarrow p, 1 \rightarrow(p \rightarrow q) \vdash_{q \mathbf{L}} 1 \rightarrow q$, (ii) $1 \rightarrow p, 1 \rightarrow(p \rightarrow q) \nvdash_{\mathbf{L}} \leq 1 \rightarrow q$, (iii) $1 \rightarrow p \forall_{\boldsymbol{q} \mathbf{L}} p$ and (iv) $1 \rightarrow p \vdash_{\mathbf{L}} \leq p$.

(4) The same counterexamples of the previous item can be used.

\section{An investigation of the logics from the viewpoint of AAL}

AAL is a mathematical framework for associating propositional logics with classes of algebras in appropriate ways. This is done by means of transfer and bridge theorems between algebraic structures and logical calculi. For a discussion, see [14] or [1].

Of the several streams that have come together to form the modern theory of AAL, one of the most prominent was initiated by Blok and Pigozzi in their seminal monograph [3]. There, the authors focus on a class of logics where the connection with algebra is especially strong (the algebraizable logics). Since then, the theory of algebraizable logics has been extended to a much wider array of logics by means of the so-called 'Leibniz heirarchy' [11], which describes how well-behaved is the relationship between a deductive system and its algebraic counterpart. At the top of this hierarchy sit the regularly algebraizable logics, those logics whose study can be almost completely reduced to the study of associated classes of algebras. At the bottom of the hierarchy lie the protoalgebraic logics, those deductive systems that do not have any purely algebraic semantics yet which have a good matrix 
semantics. For the reader unfamiliar with this approach to logic, we refer to the monograph [9] and the survey [14] (a reader interested only in the parts of this theory necessary to follow the present article can read [12] where all the necessary definitions are introduced).

The now traditional Blok-Pigozzi theory of algebraizable logics, based on matrices, has been extended to logics weaker than the protoalgebraic ones. Lying below even the protoalgebraic logics in the Leibniz hierarchy are the non-protoalgebraic logics, those deductive systems that do not possess a good matrix semantics. The study of non-protoalgebraic logics requires the theory of generalized matrices, as developed by the Barcelona group of logicians and described in the monograph [13].

Our aim in this section is to study the new logics $\boldsymbol{q} \mathbf{E}$ and $\boldsymbol{S q \mathbf { L }}$ within the framework of first- and second-order AAL. For the logics $\mathbf{E}$ and $\mathbf{L} \leq$ this has already been done in the literature (see [12, 23]). The results for the logics $\mathbf{L}$ and $\mathbf{L} \leq$ can be summarized as follows:

- if $\mathbf{A}$ is a Wajsberg algebra, then a matrix $\langle\mathbf{A}, F\rangle$ is a model of $\mathbf{E}$ iff $F$ is an implicative filter (i.e. $1 \in F$ and $F$ is closed under Modus Ponens),

- the reduced full generalized models of $\mathbf{E}$ are exactly the generalized matrices of the form $\langle\mathbf{A}, \mathcal{F}(\mathbf{A})\rangle$ where $\mathbf{A}$ is a Wajsberg algebra and $\mathcal{F}(\mathbf{A})$ is the family of implicative filters of $\mathbf{A}$,

- the reduced models of $\mathbf{E}$ are the matrices $\langle\mathbf{A}, F\rangle$, where $\mathbf{A}$ is a Wajsberg algebra and $F=\{1\}$,

- if $\mathbf{A}$ is a Wajsberg algebra, then a matrix $\langle\mathbf{A}, F\rangle$ is a model of $\mathbf{L} \leq$ iff $F$ is a lattice filter,

- the reduced full generalized models of $\mathbf{L} \leq$ are exactly the generalized matrices of the form $\left\langle\mathbf{A}, \mathcal{F}_{\leq}(\mathbf{A})\right\rangle$ where $\mathbf{A}$ is a Wajsberg algebra and $\mathcal{F}_{\leq}(\mathbf{A})$ is the family of lattice filters of $\mathbf{A}$,

- the reduced models of $\mathbf{L} \leq$ are the matrices $\langle\mathbf{A}, F\rangle$, where $\mathbf{A}$ is a Wajsberg algebra and $F$ is a lattice filter such that $\{1\}$ is the only implicative filter $G$ with the property that

$$
\forall x, y \in A(x \leftrightarrow y \in G \& x \in F \Rightarrow y \in F),
$$

- $\operatorname{Alg}^{*}(\mathbf{L})=\operatorname{Alg}(\mathbf{L})=\mathbb{W}=\operatorname{Alg}(\mathbf{L} \leq)=\operatorname{Alg}^{*}(\mathbf{L} \leq),{ }^{5}$

- the logic $\mathbf{E}$ is algebraizable with Wajsberg algebras as equivalent algebraic semantics, while $\mathbf{E} \leq$ is not protoalgebraic. ${ }^{6}$

In the next subsection and in the sequel, we will need to introduce certain classes of quasi-Wajsberg algebras, abridged in Table 4.

\subsection{Quasi-Łukasiewicz logic $\mathbf{q} \mathbf{L}$}

If $\mathbf{L}$ is any logic and $\mathbf{A}$ is an algebra of the same type, we will henceforth resort to the convention, customary in AAL, of abbreviating the expression 'deductive filter of $\mathrm{L}$ on $\mathbf{A}$ ' by ' $\mathrm{L}$-filter on $\mathbf{A}$ '.

LEMMA 31

Let $\mathbf{A}$ be a quasi-Wajsberg algebra, and $F \subseteq A$. Then, $F$ is a $q \mathbf{L}$-filter on $\mathbf{A}$ iff the following conditions hold:

(1) $1 \in F$,

\footnotetext{
${ }^{5}$ We remind the reader that the classes $\boldsymbol{A l g}{ }^{*}(\boldsymbol{L})$ and $\boldsymbol{A l g}(\boldsymbol{L})$ are essentially the classes of reduced algebras associated with the logic $L$ using, respectively, matrices and generalized matrices. As has been pointed out above, for a detailed definition of these classes we refer the reader to [13].

${ }^{6}$ The proof given in [12, Theorem 3.11] uses a complex algebraic construction and can be considerably simplified. It is enough to check that in Chang's algebra (see [7, p. 474]) the Leibniz operator is not monotone over lattice filters, and this is easily checked.
} 
TABLE 4. Strict inclusions among the classes of algebras introduced

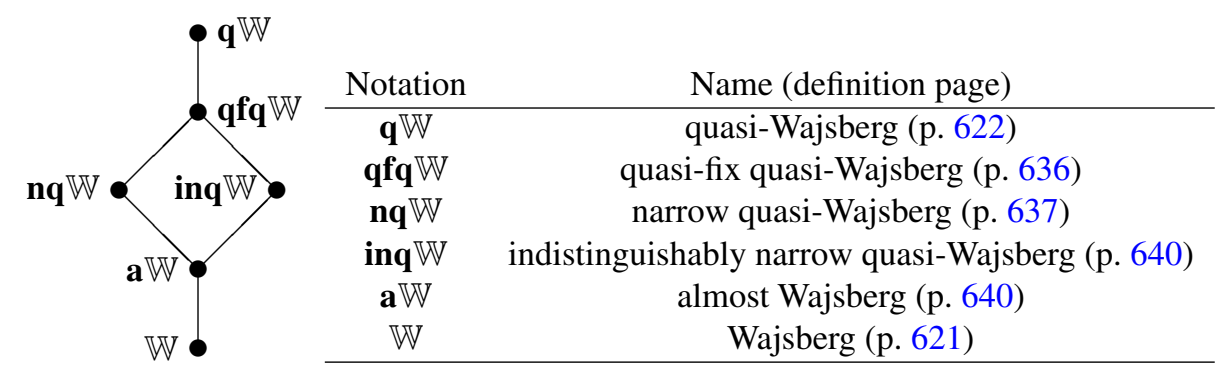

(2) if $a \leq b$ and $a \in F$ then $1 \rightarrow b \in F$,

(3) if $a, b \in F$ then $a \odot b \in F$,

(4) if $0 \in F$ and $a \in F$, then $\neg a \in F$.

Proof. Assume that $F$ is a $\boldsymbol{q} \mathbf{L}$-filter on $\mathbf{A}$. This means that $F$ is closed under the rules of $\mathbf{E}$. Then, (i) axiom (A5) yields Condition 1, (ii) $p \rightarrow q, p \vdash_{q \mathbf{L}} 1 \rightarrow q$ gives Condition 2, (iii) $p, q \vdash_{q \mathbf{L}} p \odot q$ gives Condition 3 and (iv) rule (Flat) suffices for Condition 4.

For the other direction, assume that $F$ satisfies these four conditions. We have to check that $F$ is closed under all axioms and rules in the calculus of Table 3 . This follows from the algebraic properties of quasi-Wajsberg algebras listed above. As an example we notice that the closure of $F$ under the rule $(\mathrm{qMP})$ is a consequence of the fact that

$$
((1 \rightarrow x) \odot(1 \rightarrow(x \rightarrow y))) \rightarrow y \approx 1
$$

holds in all quasi-Wajsberg algebras (use Corollary 14).

\section{DEFINITION 32}

Given a quasi-Wajsberg algebra $\mathbf{A}$, a set $F \subseteq A$ satisfying all the four properties of Lemma 31 will be called a quasi-implicative filter. The family of quasi-implicative filters of $\mathbf{A}$ will be denoted by $\mathcal{F}_{q}(\mathbf{A})$.

Observe that, if $\mathbf{A}$ is flat, the quasi-implicative filters of $\mathbf{A}$ are exactly the universes of its subalgebras.

In the following remark we calculate the Leibniz congruence of any quasi-implicative filter of a quasi-Wajsberg algebra. The key is the representation stated in Theorem 8.

REMARK 33 (Leibniz congruences of $\boldsymbol{q} \mathbf{L}$-filters)

Let $\mathbf{A}$ be an arbitrary quasi-Wajsberg algebra. By Theorem 8 we know that there is a flat quasiWajsberg algebra $\mathbf{F}$ such that $\mathbf{A} \leq \mathbf{F} \times \mathbf{R}(\mathbf{A})$. It is a simple exercise to check that the quasi-implicative filters of $\mathbf{A}$ are precisely the subsets $F \subseteq A$ satisfying one (and only one) of the following conditions (here $\biguplus$ denotes disjoint union):

(filt1) $F=F^{\prime} \biguplus X$ where $F^{\prime}$ is a proper implicative filter of $\mathbf{R}(\mathbf{A}), X \subseteq A, X \cap R(A)=\emptyset$ and $R(X) \subseteq F^{\prime}$ (i.e. if $x \in X$ then $1 \rightarrow x \in F^{\prime}$ ),

(filt2) $F=R(A) \biguplus X$ where $X \subseteq A, X \cap R(A)=\emptyset$ and $X$ is closed under negation (i.e. if $x \in X$ then $\neg x \in X)$. 
We can now calculate the Leibniz congruence $\boldsymbol{\Omega}_{\mathbf{A}}(F)$ for any quasi-implicative filter $F$ using this representation and the characterization of the Leibniz congruence as the largest congruence compatible with $F[3]$. We get that

- if $F$ is of the form (filt1), then $\boldsymbol{\Omega}_{\mathbf{A}}(F)$ is the congruence given by the following definition

$$
\langle a, b\rangle \in \boldsymbol{\Omega}_{\mathbf{A}}(F) \quad \text { iff } \quad\left\{\begin{array}{l}
a \leftrightarrow b \in F^{\prime} \\
a \in F \Leftrightarrow b \in F \\
\neg a \in F \Leftrightarrow \neg b \in F
\end{array}\right.
$$

- if $F$ is of the form (filt2), then $\mathbf{\Omega}_{\mathbf{A}}(F)$ is the congruence whose cosets are $F$ and $A \backslash F$.

Now that we know the behaviour of the Leibniz congruences, we are ready to characterize the reduced full generalized models of $\boldsymbol{q} \mathbf{\mathbf { L }}$. A crucial role in the characterization is played by the class of quasi-Wajsberg algebras satisfying the quasi-fix quasiequation

$$
1 \rightarrow x \approx \neg(1 \rightarrow x) \quad \Rightarrow \quad x \approx \neg x
$$

which says that the median cloud of an algebra in this class (in the terminology of [20]), if there is any such, consists exclusively of fixpoints under negation. We denote this class by qfq $\mathbb{W}$ and its members will be called quasi-fix quasi-Wajsberg algebras. Examples of quasi-fix quasi-Wajsberg algebras are as follows: i) all Wajsberg algebras; ii) the Sand Glass (cf. (qW37) and Example 4 of [20]); and iii) the algebras in $\mathbf{V}\left(\mathbf{F}_{10}\right)$, i.e. all flat algebras satisfying $x \approx \neg x$.

It is not hard to prove that $\mathbf{q f q} \mathbb{W}$ is not a variety, because it is not closed under quotients. ${ }^{7}$ However, the intersection of $\mathbf{q f q} \mathbb{W}$ with the class of flat quasi-Wajsberg algebras is $\mathbf{V}\left(\mathbf{F}_{\mathbf{1 0}}\right)$. Finally, we point out that the variety generated by $\mathbf{q f q} \mathbb{W}$ is the class $\mathbf{q} \mathbb{W}$ of quasi-Wajsberg algebras. ${ }^{8}$

PROPOSITION 34

The following conditions are equivalent:

(1) $\langle\mathbf{A}, \mathcal{C}\rangle$ is a reduced full generalized model of $\boldsymbol{q} \mathbf{L}$,

(2) $\mathbf{A}$ is a member of $\mathbf{q f q} \mathbb{W}$ and $\mathcal{C}=\mathcal{F}_{q}(\mathbf{A})$.

Proof. $1 \Rightarrow 2$ ): First of all let us see that $\mathbf{A}$ is a quasi-Wajsberg algebra. By Theorem 21 we know that every equation $\alpha \approx \beta$ satisfied by quasi-Wajsberg algebras is such that

$$
\text { for every formula } \delta(p, \vec{q}) \text {, it holds that } \delta(p / \alpha, \vec{q}) \vdash_{\boldsymbol{q} \mathbf{E}} \delta(p / \beta, \vec{q}) \text {. }
$$

On the other hand, the general theory of Leibniz congruences tells us that

$$
\begin{array}{ll}
\langle a, b\rangle \in \mathbf{\Omega}_{\mathbf{A}}(F) \quad \text { iff } & \begin{array}{l}
\text { for every formula } \delta(p, \vec{q}) \text { and every finite sequence } \vec{c} \text { in } A, \\
\text { it holds that: } \delta^{\mathbf{A}}(a, \vec{c}) \in F \Longleftrightarrow \delta^{\mathbf{A}}(b, \vec{c}) \in F .
\end{array}
\end{array}
$$

Using the last two sentences we conclude that for every equation $\alpha \approx \beta$ satisfied by quasiWajsberg algebras and every homomorphism $h$ from the algebra of formulas into $\mathbf{A}$ it holds that $\langle h(\alpha), h(\beta)\rangle \in \bigcap\left\{\boldsymbol{\Omega}_{\mathbf{A}}(F): F \in \mathcal{C}\right\}$. Since our generalized model is reduced we get that $h(\alpha)=h(\beta)$.

\footnotetext{
${ }^{7}$ In fact, factoring the Sand Glass by the congruence $\tau$ (see [20]) yields the algebra $\mathbf{F}_{\mathbf{0 2}}$, which is not quasi-fix.

${ }^{8}$ By [6, Proof of Lemma 38] it is known that $\mathbf{F}_{\mathbf{1 2}}$ generates the variety of flat quasi-Wajsberg algebras (indeed, by [6, Lemma 10] it is enough to consider its subalgebra $\mathbf{F}_{\mathbf{0 2}}$ ). Hence, by Corollary 9 the class $\left\{\mathbf{S t}, \mathbf{F}_{\mathbf{1 2}}\right\}$ generates the variety of quasi-Wajsberg algebras.
} 
Hence, all equations satisfied by quasi-Wajsberg algebras are also satisfied by $\mathbf{A}$. Therefore, $\mathbf{A}$ is a quasi-Wajsberg algebra.

Next we are going to see that the quasi-fix quasiequation is satisfied by $\mathbf{A}$. If not, there is an element $a \in A$ such that $1 \rightarrow a=\neg(1 \rightarrow a)$ and $a \neq \neg a$. By the characterization of Leibniz congruences given in Remark 33, it is very easy ${ }^{9}$ to check that $\langle a, \neg a\rangle \in \mathbf{\Omega}_{\mathbf{A}}(F)$ for every quasi-implicative filter $F$. Thus, $\langle a, \neg a\rangle \in \bigcap\left\{\boldsymbol{\Omega}_{\mathbf{A}}(F): F \in \mathcal{C}\right\}$ and $a \neq \neg a$. This contradicts the fact that the generalized model is reduced. So the quasi-fix quasiequation is satisfied by $\mathbf{A}$.

Since we are considering a reduced full generalized model we know that $\mathcal{C}$ is the family of filters of the logic $q \mathbf{L}$ in $\mathbf{A}$. By Lemma 31 it follows that $\mathcal{C}=\mathcal{F}_{q}(\mathbf{A})$.

$2 \Rightarrow 1)$ : Let us assume that $\mathbf{A} \in \mathbf{q f q} \mathbb{W}$ and $\mathcal{C}=\mathcal{F}_{q}(\mathbf{A})$. By Lemma 31 we know that $\langle\mathbf{A}, \mathcal{C}\rangle$ is a full generalized model of $\boldsymbol{q} \mathbf{E}$. We only need to prove that it is reduced, i.e. $\bigcap\left\{\boldsymbol{\Omega}_{\mathbf{A}}(F): F \in \mathcal{F}_{q}(\mathbf{A})\right\}$ is the identity relation. Thus, let us consider $a, b \in A$ with $a \neq b$. We are going to prove that $\langle a, b\rangle \notin$ $\bigcap\left\{\boldsymbol{\Omega}_{\mathbf{A}}(F): F \in \mathcal{C}\right\}$ by a case-splitting argument.

If it is not the case that $a \chi b$ : since $a \leftrightarrow b \neq 1$, Remark 33 tells us that $\langle a, b\rangle \notin \mathbf{\Omega}_{\mathbf{A}}(\{1\})$; however, $\{1\}$ is a quasi-implicative filter.

If $a \chi b$ with one (and only one) of them regular: We define $F$ as the quasi-implicative filter of type (filt2) given by $R(A) \biguplus \emptyset$. By Remark 33 it follows that $\langle a, b\rangle \notin \mathbf{\Omega}_{\mathbf{A}}(F)$.

If $a \chi b$ with $a$ and $b$ non-regular: first of all we remark that thanks to (qfix) it holds that $b \neq \neg a$ (because if $b=\neg a$ then $1 \rightarrow a=\neg(1 \rightarrow a)$ and $a \neq \neg a)$. We define $F$ as the quasi-implicative filter of type (filt2) given by $R(A) \biguplus\{a, \neg a\}$. Then, since $a \in F$ and $b \notin F$ by Remark 33 it follows that $\langle a, b\rangle \notin \mathbf{\Omega}_{\mathbf{A}}(F)$.

In order to characterize the reduced models of $\boldsymbol{q} \mathbf{E}$ we need to introduce another class of algebras. A quasi-Wajsberg algebra $\mathbf{A}$ is a narrow quasi-Wajsberg algebra if it satisfies the following first-order properties:

- if $a \in R(A) \backslash\{0,1\}$, then the cardinality of $a / \chi$ is 1 and

- the cardinality of $1 / \chi$ is at most 2 .

We will denote by nq $\mathbb{W}$ the family of narrow quasi-Wajsberg algebras; examples include: (i) all Wajsberg algebras; (ii) the Sand Glass; (iii) $\mathbf{F}_{\mathbf{1 0}}$, but no other flat algebra in $\mathbf{V}\left(\mathbf{F}_{\mathbf{1 0}}\right)$. It is obvious that $\mathbb{W} \subseteq \mathbf{n q} \mathbb{W} \subseteq \mathbf{q f q} \mathbb{W}$. The class of narrow quasi-Wajsberg algebras is not closed under direct products. ${ }^{10}$ Later on, as a consequence of the general theory of AAL, we will see that the variety generated by $\mathbf{n q} \mathbb{W}$ is the class of quasi-Wajsberg algebras.

\section{PROPOSITION 35}

The following conditions are equivalent:

(1) $\langle\mathbf{A}, F\rangle$ is a reduced model of $\boldsymbol{q} \mathbf{L}$,

(2) $\mathbf{A}$ is a member of $\mathbf{n q} \mathbb{W}$ and $F=\{1\}$.

ProOF. $1 \Rightarrow 2$ ): Let us assume that $\langle\mathbf{A}, F\rangle$ is a reduced model of $\boldsymbol{q} \mathbf{E}$. Then $\langle\mathbf{A}, \mathcal{C}\rangle$, with $\mathcal{C}$ the family of $\boldsymbol{q} \mathbf{L}$-filters on $\mathbf{A}$, is a reduced full generalized model of $\boldsymbol{q} \mathbf{L}$. By Proposition 34 it follows that $\mathbf{A} \in \mathbf{q f q} \mathbb{W}$

\footnotetext{
${ }^{9}$ We note that if $a$ belongs to a quasi-implicative filter $F$ then $0 \in F$ because $0=(1 \rightarrow a) \odot \neg(1 \rightarrow a)=(1 \rightarrow a) \odot(1 \rightarrow a) \in F$. And the same holds in case that $\neg a \in F$ (remember that $\neg(1 \rightarrow a)=1 \rightarrow \neg a$ ). Using these two statements and Condition (4) in the definition of quasi-implicative filters, it happens that (i) if $F$ is a quasi-implicative filter of type (filt1) then $\langle a, \neg a\rangle \in \boldsymbol{\Omega}_{\mathbf{A}}(F)$ and (ii) if $F$ is a quasi-implicative filter of type (filt2) then $\langle a, \neg a\rangle \in \mathbf{\Omega}_{\mathbf{A}}(F)$.

${ }^{10}$ Consider any non-trivial power of $\mathbf{F}_{\mathbf{1 0}}$.
} 
and that $F$ is a quasi-implicative filter. In order to prove the remaining conditions we distinguish two cases.

Case $F$ is of type (filt1): By Remark 33 and the fact that $\boldsymbol{\Omega}_{\mathbf{A}}(F)=I d$ it is obvious that $F^{\prime}$ is the proper implicative filter $\{1\}$ of $\mathbf{R}(\mathbf{A})$. Since $R(X) \subseteq F^{\prime}$ and $1 \notin X$ we know that $F=\{1\} \biguplus X \subseteq 1 / \chi$. Then, it is clear that all elements in $F$ are related by $\boldsymbol{\Omega}_{\mathbf{A}}(F)$ and that all elements in $(1 / \chi) \backslash F$ are also related by $\boldsymbol{\Omega}_{\mathbf{A}}(F)$. Using that $\boldsymbol{\Omega}_{\mathbf{A}}(F)=I d$ it follows that both $F$ and $(1 / \chi) \backslash F$ have cardinality at most 1 . This implies that $F=\{1\}$ and that $1 / \chi$ has cardinality at most 2 . Thus, the only remaining condition we have to prove is that if $a \in R(A) \backslash\{0,1\}$, then the cardinality of $a / \chi$ is 1 . If not, there are two elements $a, b \in A$ such that $a \neq b$ and $1 \rightarrow a=1 \rightarrow b \notin\{0,1\}$. Since $F \subseteq 1 / \chi$ we deduce that $a \notin F, b \notin F, \neg a \notin F$ and $\neg b \notin F$. By Remark 33 this implies that $\langle a, b\rangle \in \boldsymbol{\Omega}_{\mathbf{A}}(F)$, contradicting the fact that $\boldsymbol{\Omega}_{\mathbf{A}}(F)=I d$.

Case $F$ is of type (filt2): Using Remark 33 and the fact that $\boldsymbol{\Omega}_{\mathbf{A}}(F)=I d$ it follows that both $F$ and $A \backslash F$ have cardinality at most 1 . Hence, $|A| \leq 2$ and $F=\{1\}$. Using that $|A| \leq 2$ it is obvious that $\mathbf{A} \in \mathbf{n q} \mathbb{W}$.

$2 \Rightarrow 1)$ : Assume that $\mathbf{A} \in \mathbf{n q} \mathbb{W}$ and $F=\{1\}$. We have to prove that $\boldsymbol{\Omega}_{\mathbf{A}}(F)=I d$. Without loss of generality we can assume that $\mathbf{A}$ is non-trivial, i.e. $|A| \geq 2$. It is clear that $F$ is a quasi-implicative filter of type (filt1). Moreover, by Remark 33 we know that

$$
\langle a, b\rangle \in \mathbf{\Omega}_{\mathbf{A}}(\{1\}) \quad \text { iff } \quad\left\{\begin{array}{l}
a \leftrightarrow b=1 \\
a=1 \Leftrightarrow b=1 \\
\neg a=1 \Leftrightarrow \neg b=1
\end{array}\right.
$$

From this characterization together with the fact that $\mathbf{A} \in \mathbf{n q} \mathbb{W}$ we obtain that $\boldsymbol{\Omega}_{\mathbf{A}}(F)=I d$.

COROLLARY 36

$\boldsymbol{A} \lg (\boldsymbol{q} \mathbf{L})=\mathbf{q f q} \mathbb{W}$ and $\boldsymbol{A} \boldsymbol{l g}^{*}(\boldsymbol{q} \mathbf{E})=\mathbf{n q} \mathbb{W}$. Moreover $\mathbf{q f q} \mathbb{W}$ and $\mathbf{n q} \mathbb{W}$ generate the same variety, which is the class of quasi-Wajsberg algebras.

Proof. The statement $\operatorname{Alg}(\boldsymbol{q} \mathbf{L})=\mathbf{q f q} \mathbb{W}$ is a consequence of Proposition 34. On the other hand, $\operatorname{Alg}^{*}(q \mathbf{L})=\mathbf{n q \mathbb { W }}$ follows from Proposition 35. From the general theory of AAL it is known that $\boldsymbol{A} \boldsymbol{l g}(\boldsymbol{q} \mathbf{L})$ and $\boldsymbol{A l g ^ { * }}(\boldsymbol{q} \mathbf{L})$ always generate the same variety [13]. We remind the reader that in Footnote 8 we pointed out that the variety generated by $\mathbf{q} \mathbf{q} \mathbf{W} \mathbb{W}$ is the class of quasi-Wajsberg algebras.

COROLLARY 37

$\vdash_{\mathbf{n q} \mathbb{W}}=\vdash_{\mathbf{q f q} \mathbb{W}}=\vdash_{\mathbf{q} \mathbb{W}}$.

PROOF. We confine ourselves to $\vdash_{\mathbf{n q} \mathbb{W}}=\vdash_{\mathbf{q} \mathbb{W}}$ (the remainder of the proof is analogous). The general theory of AAL implies that $\boldsymbol{q} \mathbf{L}$ is complete with respect to its class of reduced models. Since $\{\langle\mathbf{A},\{1\}\rangle$ : $\mathbf{A} \in \mathbf{n q} \mathbb{W}\}$ is a class of models of $\boldsymbol{q} \mathbf{L}$ (see Lemma 31) which contains all reduced models of $\boldsymbol{q} \mathbf{L}$ (see Proposition 35) we know that $\{\langle\mathbf{A},\{1\}\rangle: \mathbf{A} \in \mathbf{n q} \mathbb{W}\}$ is complete with respect to $q \mathbf{L}$. By the definition of $\vdash_{\mathbf{n q} \mathbb{W}}$, this is equivalent to $\vdash_{\mathbf{n q} \mathbb{W}}=\vdash_{\mathbf{q} \mathbb{W}}$.

We finally proceed to place $\boldsymbol{q} \mathbf{E}$ within the Leibniz hierarchy. To show that a logic is not protoalgebraic it is necessary and sufficient to check that there is an algebra $\mathbf{A}$ such that the Leibniz operator is not monotone over the deductive filters of the logic on A. In most of the cases found in the literature this is the standard method to prove that a logic fails to be protoalgebraic. In this case we can apply this method ${ }^{11}$ as well as a different proof that uses the characterization of Proposition 20.

\footnotetext{
${ }^{11}$ Hint: One option is to take $\mathbf{A}$ as the flat quasi-Wajsberg algebra $\mathbf{F}_{\mathbf{1 2}}$. Another possibility is to take $\mathbf{A}=\mathbf{S}$ and check that $\boldsymbol{\Omega}_{\mathbf{A}}(\{(1,1 / 2)\}) \not \boldsymbol{\Omega}_{\mathbf{A}}(\{(1,1 / 2),(0,1)\})$. This last possibility gives us another proof of Proposition 46 in the sequel.
} 


\section{PROPOSITION 38}

$\boldsymbol{q} \mathbf{E}$ is not protoalgebraic.

Proof. We have to see that there is no set $\Delta(p, q)$ of formulas with two variables satisfying $\emptyset \vdash_{q \mathbf{L}}$ $\Delta(p, p)$ and $p, \Delta(p, q) \vdash_{q \mathbf{L}} q$. Let us assume that this set exists. Then, by Theorem 21, Proposition 20 and $p, \Delta(p, q) \vdash_{q \mathbf{E}} q$ we know that at least one of the following conditions holds:

- $\left\{q, \neg^{2} q, \neg^{4} q, \ldots\right\} \cap \Delta(p, q) \neq \emptyset$ : This is a contradiction with $\emptyset \vdash_{q \mathbf{L}} \Delta(p, p)$.

- $\left\{\neg q, \neg^{3} q, \neg^{5} q, \ldots\right\} \cap \Delta(p, q) \neq \emptyset$ and $p, \Delta(p, q) \vdash_{q \mathbf{L}} 0$ : This is also a contradiction with $\emptyset \vdash_{q \mathbf{E}}$ $\Delta(p, p)$.

\subsection{Square Quasi-Łukasiewicz logic SqL}

LEMMA 39

Let $\mathbf{A}$ be a quasi-Wajsberg algebra, and $F \subseteq A$. Then, $F$ is a $S q \mathbf{L}$-filter on $\mathbf{A}$ iff the following conditions hold:

(1) $F$ is a quasi-implicative filter,

(2) if $0 \in F$ then $F=A$.

Proof. This is a consequence of the fact that $S \boldsymbol{q} \mathbf{L}$ is obtained from $\boldsymbol{q} \mathbf{\mathbf { L }}$ by adding the rule (EFQ).

DEFINITION 40

Given a quasi-Wajsberg algebra $\mathbf{A}$, a set $F \subseteq A$ satisfying all the previous properties will be called a square quasi-implicative filter. The family of square quasi-implicative filters of $\mathbf{A}$ will be denoted by $\mathcal{F}_{s q}(\mathbf{A})$.

We point out that in non-trivial flat algebras the set $\{1\}$ is not a square quasi-implicative filter. This is a difference with quasi-implicative filters because in any quasi-Wajsberg algebra the set $\{1\}$ is a quasi-implicative filter.

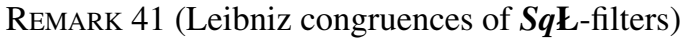

First of all we recall that the Leibniz congruence $\boldsymbol{\Omega}_{\mathbf{A}}(F)$ of a square quasi-implicative filter $F$ is an absolute notion in the sense that it only depends on the algebra $\mathbf{A}$ and not on the logic $S q \mathbf{L}$. Hence, the calculations for the Leibniz congruences of Remark 33 are valid also in this case; the only difference is in the family of filters. Let $\mathbf{A}$ be an arbitrary quasi-Wajsberg algebra. Then, there is a flat quasi-Wajsberg algebra $\mathbf{F}$ such that $\mathbf{A} \subseteq \mathbf{F} \times \mathbf{R}(\mathbf{A})$. It is an easy exercise to check that the square quasi-implicative filters of $\mathbf{A}$ are precisely the subsets $F \subseteq A$ satisfying one (and only one) of the following conditions:

(filt1) $F=F^{\prime} \biguplus X$ where $F^{\prime}$ is a proper implicative filter of $\mathbf{R}(\mathbf{A}), X \subseteq A, X \cap R(A)=\emptyset$ and $R(X) \subseteq F^{\prime}$ (i.e. if $x \in X$ then $1 \rightarrow x \in F^{\prime}$ ),

(filt3) $F=A$.

From Remark 33 and the previous discussion we get that

- if $F$ is of the form (filt1), then $\boldsymbol{\Omega}_{\mathbf{A}}(F)$ is the congruence given by the following definition

$$
\langle a, b\rangle \in \boldsymbol{\Omega}_{\mathbf{A}}(F) \quad \text { iff } \quad\left\{\begin{array}{l}
a \leftrightarrow b \in F^{\prime} \\
a \in F \Leftrightarrow b \in F \\
\neg a \in F \Leftrightarrow \neg b \in F
\end{array}\right.
$$


- if $F$ is of the form (filt3), then $\boldsymbol{\Omega}_{\mathbf{A}}(F)=A \times A$.

Next we introduce the last two classes of algebras that we will need. We will denote by inq $\mathbb{W}$ the class of quasi-Wajsberg algebras satisfying the following condition:

if $a \in R(A)$ is such that $a$ and $\neg a$ are exactly in the same implicative filters of the Wajsberg algebra $\mathbf{R}(\mathbf{A})$, then the cardinality of $a / \chi$ is 1 .

We call the members of this class indistinguishably narrow quasi-Wajsberg algebras. Examples include: (i) all Wajsberg algebras and (ii) the Sand Glass. No non-trivial flat algebra is in this class. It is obvious that $\mathbb{W} \subseteq$ inq $\mathbb{W} \subseteq \mathbf{q f q} \mathbb{W}$. It is also clear that the following conditions are equivalent:

- $a$ and $\neg a$ are exactly in the same implicative filters;

- the implicative filter generated by $a$ coincides with the implicative filter generated by $\neg a$.

Using the well known characterization of the implicative filter generated by a set in the case of Wajsberg algebras it is obvious that the condition defining indistinguishably narrow quasi-Wajsberg algebras is equivalent to the following family of quasiequations $(n \in \omega)$ :

$$
(x \odot \stackrel{n}{2} \odot x) \rightarrow \neg x \approx 1 \&(\neg x \odot \stackrel{n}{ } \odot \neg \neg) \rightarrow x \approx 1 \& x \approx 1 \rightarrow y \quad \Rightarrow \quad x \approx y
$$

Therefore, inq $\mathbb{W}$ is a quasiequational class. However, it is not closed under quotients. ${ }^{12}$ We point out that the variety generated by inq $\mathbb{W}$ is the class of all quasi-Wajsberg algebras. ${ }^{13}$

The class of almost Wajsberg algebras, denoted by $\mathbf{a} \mathbb{W}$, is defined as the class of quasi-Wajsberg algebras satisfying

- if $a \in R(A) \backslash\{0,1\}$, then the cardinality of $a / \chi$ is 1 ,

- the cardinality of $1 / \chi$ is at most 2 ,

- if $\mathbf{A}$ is flat then it is trivial.

It is obvious that $\mathbf{a} \mathbb{W}=\mathbf{n q} \mathbb{W} \cap \mathbf{i n q} \mathbb{W}$. It is also obvious that the class of almost Wajsberg algebras is definable by a first-order sentence. Hence, it is closed under ultraproducts. However, it is not closed under products. ${ }^{14}$

Proposition 42

The following conditions are equivalent:

(1) $\langle\mathbf{A}, \mathcal{C}\rangle$ is a reduced full generalized model of $\boldsymbol{S q \mathbf { L }}$,

(2) $\mathbf{A}$ is a member of inq $\mathbb{W}$ and $\mathcal{C}=\mathcal{F}_{s q}(\mathbf{A})$.

Proof. $1 \Rightarrow 2$ ): As a consequence of Proposition 34 and Lemma 39 we only need to prove that the condition defining indistinguishably narrow quasi-Wajsberg algebras holds. Let us assume that $b \chi a \in R(A)$ is such that $a$ and $\neg a$ are exactly in the same implicative filters of the Wajsberg algebra $\mathbf{R}(\mathbf{A})$ and that $a \chi b$. We have to check that $a=b$. Since $\langle\mathbf{A}, \mathcal{C}\rangle$ is reduced and $\mathcal{C}=\mathcal{F}_{s q}(\mathbf{A})$ it is enough to prove that $\langle a, b\rangle \in \bigcap\left\{\boldsymbol{\Omega}_{\mathbf{A}}(F): F \in \mathcal{F}_{s q}(\mathbf{A})\right\}$. Thus, we have to prove that $\langle a, b\rangle$ belongs to any congruence compatible with a square quasi-implicative filter $F$. If $F$ is of type (filt3) then it is obvious that $\langle a, b\rangle \in \mathbf{\Omega}_{\mathbf{A}}(F)$. If $F$ is of type (filt1), then $F^{\prime}$ is a proper implicative filter of $\mathbf{R}(\mathbf{A})$. Since

\footnotetext{
${ }^{12}$ Once again, consider the quotient of the Sand Glass modulo $\tau$.

${ }^{13}$ Hint: By [6, Lemma 10] it is known that $\mathbf{F}_{\mathbf{0 2}}$ generates the variety of flat quasi-Wajsberg algebras. Hence, by Corollary 9 the class $\left\{\mathbf{S t}, \mathbf{F}_{\mathbf{0 2}}\right\}$ generates the variety of quasi-Wajsberg algebras.

${ }^{14}$ Consider any non-trivial power of the Sand Glass.
} 
it is proper we know that $a \notin F^{\prime}$ and $\neg a \notin F^{\prime}$. Using that $R(X) \subseteq F^{\prime}$ it follows that $b \notin X$ and $\neg b \notin X$. Hence, none of the elements $a, \neg a, b$ and $\neg b$ belongs to $F$. Using the characterization of $\boldsymbol{\Omega}_{\mathbf{A}}(F)$ provided in Remark 41 we get that $\langle a, b\rangle \in \mathbf{\Omega}_{\mathbf{A}}(F)$.

$2 \Rightarrow 1)$ : This proof is very similar to the one given for Proposition 34 . Assume that $\mathbf{A} \in \mathbf{i n q} \mathbb{W}$ and $\mathcal{C}=\mathcal{F}_{s q}(\mathbf{A})$. By Lemma 39 we know that $\langle\mathbf{A}, \mathcal{C}\rangle$ is a full generalized model of $\boldsymbol{S q} \mathbf{L}$. We have to prove that $\bigcap\left\{\boldsymbol{\Omega}_{\mathbf{A}}(F): F \in \mathcal{F}_{s q}(\mathbf{A})\right\}$ is the identity relation. Thus, let us consider $a, b \in A$ with $a \neq b$. We check that $\langle a, b\rangle \notin \bigcap\left\{\boldsymbol{\Omega}_{\mathbf{A}}(F): F \in \mathcal{C}\right\}$ by a case-splitting argument.

If it is not the case that $a \chi b$ : Since $a \leftrightarrow b \neq 1$ Remark 41 tells us that $\langle a, b\rangle \notin \boldsymbol{\Omega}_{\mathbf{A}}(\{1\})$, and $\{1\}$ is a square quasi-implicative filter (we stress that this cannot be a flat algebra, since $\chi$ is not the universal relation).

If $a \chi b$ with one (and only one) of $a$ and $b$ regular: Let us assume that $a$ is regular. Since $a \neq b$ we know that the size of $a / \chi$ is at least 2 . Since $\mathbf{A}$ is an indistinguishably narrow quasi-Wajsberg algebra, $a$ and $\neg a$ are distinguishable using an implicative filter of $\mathbf{R}(\mathbf{A})$. Without loss of generality (because $\neg a \chi \neg b$ ) we can assume that there is a proper implicative filter $G$ such that $a \in G$ and $\neg a \notin G$. We define $F$ as the square quasi-implicative filter of type (filt1) where $F^{\prime}=G$ and $X=\emptyset$. Then, $a \in F$ while $b \notin F$. By Remark 41 it follows that $\langle a, b\rangle \notin \mathbf{\Omega}_{\mathbf{A}}(F)$.

If $a \chi b$ with $a$ and $b$ non-regular: Since $a \neq b$ we know that the size of $a / \chi$ is at least 3 . Let $c$ be the regular element $1 \rightarrow a$. Using that $\mathbf{A}$ is an indistinguishably narrow quasi-Wajsberg algebra it follows that $c$ and $\neg c$ are distinguishable using an implicative filter of $\mathbf{R}(\mathbf{A})$. Without loss of generality (because $\neg a \chi \neg b$ ) we can assume that there is a proper implicative filter $G$ such that $c \in G$ and $\neg c \notin G$. We define $F$ as the square quasi-implicative filter of type (filt1) where $F^{\prime}=G$ and $X=\{a\}$. Then, $a \in F$ while $b \notin F$. By Remark 41 it follows that $\langle a, b\rangle \notin \mathbf{\Omega}_{\mathbf{A}}(F)$.

PROPOSITION 43

The following conditions are equivalent:

(1) $\langle\mathbf{A}, F\rangle$ is a reduced model of $\boldsymbol{S q} \mathbf{L}$,

(2) $\mathbf{A}$ is a member of $\mathbf{a} \mathbb{W}$ and $F=\{1\}$.

Proof. $1 \Rightarrow 2$ ): By Proposition 35 we know that $\mathbf{A} \in \mathbf{n q} \mathbb{W}$ and $F=\{1\}$, and by Proposition 42 it follows that $\mathbf{A} \in \mathbf{i n q} \mathbb{W}$. Hence, $\mathbf{A} \in \mathbf{n q} \mathbb{W} \cap$ inq $\mathbb{W}=\mathbf{a} \mathbb{W}$.

$2 \Rightarrow 1)$ : Assume that $\mathbf{A} \in \mathbf{a} \mathbb{W}$ and $F=\{1\}$. We have to prove that $\boldsymbol{\Omega}_{\mathbf{A}}(F)=I d$. Without loss of generality we can assume that $\mathbf{A}$ is non-trivial, i.e. $|A| \geq 2$. But if $\mathbf{A} \in \mathbf{a} \mathbb{W}$ is non-trivial then $\mathbf{A} \in \mathbf{n q} \mathbb{W}$. Therefore, by Proposition 35 we get that $\boldsymbol{\Omega}_{\mathbf{A}}(F)=I d$.

COROLlary 44

$\boldsymbol{A l g}(\boldsymbol{S q} \mathbf{L})=\operatorname{inq} \mathbb{W}$ and $\operatorname{Alg}^{*}(\boldsymbol{S q} \mathbf{L})=\mathbf{a} \mathbb{W}$. Moreover inq $\mathbb{W}$ and $\mathbf{a} \mathbb{W}$ generate the same variety, which is $\mathbf{q} \mathbb{W}$.

Proof. The proof is analogous to the one given for Corollary 36. We remind the reader that we pointed out above that the variety generated by inq $\mathbb{W}$ is $\mathbf{q} \mathbb{W}$.

COROLlary 45

$\vdash_{\text {inq } \mathbb{W}}=\vdash_{\mathbf{a} \mathbb{W}}=\vdash_{S q \mathbf{L}}$.

Proof. The proof is analogous to the one given for Corollary 37.

PROPOSITION 46

$S q \mathbf{L}$ is not protoalgebraic. 
Proof. The same proof that was given for Proposition 38 works also in this case (but this time using Theorem 27 and Proposition 26) except for the method to obtain a contradiction from the second case $p, \Delta(p, q) \vdash_{S q \mathbf{E}} 0$. Now, in case $p, \Delta(p, q) \vdash_{\boldsymbol{S} q \mathbf{\mathbf { L }}} 0$, substitution invariance implies that $p, \Delta(p, p) \vdash_{\boldsymbol{S q \mathbf { L }}} 0$. Using the fact that the formulas in $\Delta(p, p)$ are theorems we get that $p \vdash_{\boldsymbol{S q \mathbf { L }}} 0$, a contradiction.

\section{Conclusions}

Quasi-MV algebras are perhaps only of limited interest for quantum computation, except for their being a 'baby example' which it may be easier to cope with first, before proceeding to the investigation of richer structures. $\sqrt{1}$ quasi-MV algebras [17] are more interesting from this point of view, in that they contain the genuinely quantum operation of square root of the inverse, which provably admits of no fuzzy counterpart (see e.g. [10]). The investigation of the logics underlying this class of structures is the aim of a article which is currently under preparation.

\section{Acknowledgements}

We thank the anonymous referees for their precious suggestions.

\section{Funding}

F.B. thanks Eurocores (LOMOREVI Eurocores Project FP006/FFI2008-03126-E/FILO); Spanish Ministry of Education and Science (project MULOG2 TIN2007-68005-C04-01); Catalan Government (2009SGR-1433).

\section{References}

[1] H. Andréka, I. Németi, and I. Sain. Algebraic logic. In Handbook of Philosophical Logic, vol. 2, D. M. Gabbay and F. Guenthner, eds, 2nd edn, pp. 133-247, Kluwer Academic Publishers, 2001.

[2] J. Berman and W. J. Blok. Algebras defined from ordered sets and the varieties they generate. Order, 23, 65-88, 2006.

[3] W. J. Blok and D. Pigozzi. Algebraizable Logics, vol. 396 of Memoirs of the American Mathematical Society. American Mathematical Society, 1989.

[4] W. J. Blok and J. G. Raftery. Assertionally equivalent quasivarieties, International Journal of Algebra and Computation, 18, 589-681, 2008.

[5] F. Bou, F. Esteva, J. M. Font, A. Gil, L. Godo, A. Torrens, and V. Verdú. Logics preserving degrees of truth from varieties of residuated lattices. Journal of Logic and Computation, 19, 1031-1069, 2009.

[6] F. Bou, F. Paoli, A. Ledda, and H. Freytes. On some properties of quasi-MV algebras and $\sqrt{1}$ quasi-MV algebras. Part 2. Soft Computing, 12, 341-352, 2008.

[7] C. C. Chang. Algebraic analysis of many-valued logics. Transactions of the Americal Mathematical Society, 88, 467-490, 1958.

[8] R. Cignoli, I. M. L. D’Ottaviano, and D. Mundici. Algebraic Foundations of Many-Valued Reasoning, Vol. 7 of Trends in Logic-Studia Logica Library. Kluwer Academic Publishers, 2000. 
[9] J. Czelakowski. Protoalgebraic Logics, Vol. 10 of Trends in Logic-Studia Logica Library. Kluwer Academic Publishers, 2001.

[10] M. L. Dalla Chiara, R. Giuntini, and R. Greechie. Reasoning in Quantum Theory, Vol. 22 of Trends in Logic_Studia Logica Library. Kluwer Academic Publishers, 2004.

[11] J. M. Font. On the Leibniz congruences. In Algebraic Methods in Logic and Computer Science. C. Rauszer ed., Vol. 28, pages 17-36, Banach Centre Publications, Institute of Mathematics, Polish Academy of Sciences, 1993.

[12] J.M. Font, A. J. Gil, A. Torrens, and V. Verdù. On the infinite-valued Lukasiewicz logic that preserves degrees of truth. Archive for Mathematical Logic, 45, 839-868, 2006.

[13] J. M. Font and R. Jansana. A General Algebraic Semantics for Sentential Logics. Vol. 7 of Lecture Notes in Logic, Springer, 2009. Electronic version freely available through Project Euclid at projecteuclid.org/euclid.lnl/1235416965.

[14] J. M. Font, R. Jansana, and D. Pigozzi. A survey on abstract algebraic logic. Studia Logica, Special Issue on Abstract Algebraic Logic, Part II, 74, 13-97, 2003.

[15] J. M. Font, A. J. Rodríguez, and A. Torrens. Wajsberg algebras. Stochastica, 8, 5-31, 1984.

[16] J. Gispert and D. Mundici. MV-algebras: a variety for magnitudes with Archimedean units. Algebra Universalis, 53, 7-43, 2005.

[17] R. Giuntini, A. Ledda, and F. Paoli. Expanding quasi-MV algebras by a quantum operator. Studia Logica, 87, 99-128, 2007.

[18] R. Giuntini, A. Ledda, and F. Paoli. Categorical equivalences for $\sqrt{ }{ }^{\prime}$ quasi-MV algebras. Journal of Logic and Computation [Epub ahead of print, doi:10.1093/logcom/exn082, 22 December 2008].

[19] T. Kowalski and F. Paoli. On some properties of quasi-MV algebras and $\sqrt{1}$ quasi-MV algebras. Part 3. Reports on Mathematical Logic, forthcoming.

[20] A. Ledda, M. Konig, F. Paoli, and R. Giuntini. MV-algebras and quantum computation. Studia Logica, 82, 245-270, 2006.

[21] F. Paoli, A. Ledda, R. Giuntini, and H. Freytes. On some properties of quasi-MV algebras and $\sqrt{ }{ }^{\prime}$ quasi-MV algebras. Part I. Reports on Mathematical Logic, 44, 53-85, 2008.

[22] F. Paoli, M. Spinks, and R. Veroff. Abelian logic and logics from pointed lattice-ordered varieties. Logica Universalis, Logica Universalis, 2, 209-233, 2008.

[23] A. J. Rodríguez, A. Torrens, and V. Verdú. Łukasiewicz logic and Wajsberg algebras. Bulletin of the Section of Logic, 19, 51-55, 1990.

[24] A. Salibra. Topological incompleteness and order incompleteness of the lambda calculus. ACM Transactions in Computational Logic, 4, 379-401, 2003.

[25] M. Spinks, F. Bou, F. Paoli, A. Ledda, and R. Giuntini. Logics of quasi-MV algebras. Available at http://www.cs.unm.edu/ spinks/ (Last accessed 14 January 2010).

[26] R. Wójcicki. Theory of Logical Calculi. Basic Theory of Consequence Operations, Vol. 199 of Synthese Library. Reidel, 1988.

Received 29 July 2008 